UG-12-35

AEI-2012-055

\title{
Supersymmetric Domain Walls
}

\author{
Eric A. Bergshoeff ${ }^{1}$, Axel Kleinschmidt ${ }^{2}$ and Fabio Riccioni ${ }^{3}$ \\ ${ }^{1}$ Centre for Theoretical Physics, University of Groningen, \\ Nijenborgh 4, 9747 AG Groningen, The Netherlands \\ email: E.A.Bergshoefforug.nl \\ ${ }^{2}$ Max Planck Institute for Gravitational Physics, Albert Einstein Institute \\ Am Muhlenberg 1, 14476 Potsdam, Germany \\ 83 \\ International Solvay Institutes \\ Campus Plaine C.P. 231, Boulevard du Triomphe, 1050 Bruxelles, Belgium \\ email: axel.kleinschmidt@aei.mpg.de \\ ${ }^{3}$ INFN Sezione di Roma, Dipartimento di Fisica, \\ Università di Roma "La Sapienza", \\ Piazzale Aldo Moro 2, 00185 Roma, Italy \\ email: Fabio.Riccioni@roma1.infn.it
}

\begin{abstract}
We classify the half-supersymmetric "domain walls", i.e. branes of codimension one, in toroidally compactified IIA/IIB string theory and show to which gauged supergravity theory each of these domain walls belong. We use as input the requirement of supersymmetric Wess-Zumino terms, the properties of the $E_{11}$ Kac-Moody algebra and the embedding tensor formalism. We show that the number of half-supersymmetric domain walls is a multiple of the number of corresponding central charges in the supersymmetry algebra, where the multiplicity is related to the degeneracy of the BPS conditions.
\end{abstract}




\section{Contents}

1 Introduction 1

2 Classifying Supersymmetric Domain Walls 8

2.1 Domain walls and supersymmetric WZ terms . . . . . . . . . 9

2.1.1 Example: $D=7$ defect branes . . . . . . . . . . . . 10

2.1.2 Example: $D=7$ domain walls . . . . . . . . . . . . 11

2.2 Supersymmetric domain walls and $E_{11}$. . . . . . . . . . . 13

3 Domain Walls and Gauged Supergravity 14

3.1 The nine-dimensional gaugings . . . . . . . . . . . . . 16

3.2 The eight-dimensional gaugings . . . . . . . . . . . . . 17

3.3 The seven-dimensional gaugings . . . . . . . . . . . . . . 19

4 Domain-wall Solutions $\quad 22$

4.1 Domain-wall solutions and supergravity . . . . . . . . . . . . 22

4.2 Domain-wall solutions and $E_{11} \ldots \ldots . \ldots . . \ldots 25$

5 Domain Walls and Central Charges 28

5.1 One central charge, many domain walls . . . . . . . . . . 28

5.2 BPS conditions and $E_{11} \ldots \ldots \ldots 33$

6 Conclusions $\quad 35$ 


\section{Introduction}

Domain walls are branes of codimension one, i.e. they have a single transverse direction. They play an important role in a wide range of situations such as setting up brane-world scenarios [1] and describing the renormalization group flow in an AdS/CFT setting [2]. A distinguishing feature of domain walls is that their existence within a supergravity context, unlike that of other branes, requires the use of a deformed supergravity theory where the deformation parameter can be a mass parameter ("massive" supergravity) or a gauge coupling constant ("gauged" supergravity). These deformed supergravity theories generically contain a potential for the scalar fields which is needed to realize the domain-wall solutions. The same potential is also needed to allow interesting cosmological solutions. The study of these cosmological solutions is relevant for our efforts to extract an expanding de Sitter solution out of a string theory compactification. Domain walls and cosmologies are related to each other via the "domain-wall/cosmology" correspondence [3]. In view of the above remarks it is important to classify all supersymmetric domain walls and determine which deformed supergravity theory they are related to.

The prime example of a half-supersymmetric domain wall is the D8-brane of IIA string theory. This brane is electrically charged with respect to the Ramond-Ramond (RR) 9-form potential $C_{9}$, thus leading to a solution such that the corresponding 10-form field strength $G_{10}$ is non-vanishing and proportional to a mass parameter $m$ (that is constant by virtue of the Bianchi identity). This means that the presence of the D8-brane source induces a cosmological constant in the theory, and this corresponds to a domain-wall solution of the Romans IIA theory [4], whose explicit form, in Einstein frame, is $[5]$

$$
\begin{aligned}
d s^{2} & =H^{9 / 8} d y^{2}+H^{1 / 8} d x^{\mu} d x^{\nu} \eta_{\mu \nu}, \\
e^{\phi} & =H^{5 / 4}, \\
C_{01 \cdots 8} & = \pm H^{-1}, \quad \text { or } \quad m= \pm H^{\prime} .
\end{aligned}
$$

Here $y$ indicates the transverse direction of the domain wall, the prime indi-

cates a differentiation with respect to $y$ and $H(y)$ is a harmonic function of $y$. The Romans deformation is a massive deformation and not a gauge defor- 
mation, ${ }^{1}$ which stems from the RR 1 -form $C_{1}$ transforming with a shift, proportional to $m$, under the gauge parameter $\Sigma_{1}$ of the Neveu-Schwarz/NeveuSchwarz (NS-NS) 2-form $B_{2}$. Therefore, $C_{1}$ is "eaten up" by $B_{2}$ and the two potentials $\left(C_{1}, B_{2}\right)$ together form a so-called Stückelberg pair describing a massive 2-form. A similar Stückelberg mechanism happens for the dual potentials $\left(D_{6}, C_{7}\right)$ where $D_{6}$ is eaten up by $C_{7}$. The RR 3 -forms $C_{3}$ and its dual 5 -form $C_{5}$ remain massless. The situation is summarized in Table $1 .^{2}$ The deformation also induces a change in the Hodge duality relations for the massive forms. They take on the form of massive duality relations that are roughly of the form $d B_{2}=m * C_{7}$. That is, the rank of dual forms is shifted by one for massive fields as compared to massless duality.

\begin{tabular}{|c||c|c|c|c|c|c|}
\hline gauging & $C_{1}$ & $B_{2}$ & $C_{3}$ & $C_{5}$ & $D_{6}$ & $C_{7}$ \\
\hline$m$ & gauged & massive & massless & massless & gauged & massive \\
\hline
\end{tabular}

Table 1: The Romans deformation in ten dimensions corresponds to a minimal gauging, leading to the elementary D8-brane domain-wall solution of IIA supergravity. "Gauged" means "eaten up" by the neighbouring form to the right.

The existence of the D8-brane in IIA string theory can be anticipated from the potentials of the massless IIA supergravity and their gauge transformations. At leading order the D8-brane couples to the pull-back of the RR 9-form potential $C_{9}$ via a Wess-Zumino (WZ) term. This term by itself is not gauge-invariant because $C_{9}$ not only transforms into a total derivative under its own gauge transformation but it also transforms to the curvature $H_{3}=d B_{2}$ of $B_{2}$ :

$$
\delta C_{9}=d \lambda_{8}+H_{3} \lambda_{6} .
$$

Moreover, the only worldvolume field introduced so far is the single embedding scalar corresponding to the transverse direction of the domain wall.

\footnotetext{
${ }^{1}$ The $\mathbb{R}^{+}$-scaling symmetry of the theory cannot be gauged since the RR 1 -form $C_{1}$ has a non-zero weight under this symmetry.

${ }^{2}$ We have not indicated the RR 9-form $C_{9}$ in Table 1 since it does not describe a physical degree of freedom. In fact, the dual of its curvature is proportional to the mass parameter $m$. We have neither indicated the two 10-form potentials that can be added to the IIA supergravity multiplet [6]. They do not couple to half-supersymmetric space-filling branes.
} 
This scalar by itself does not fill a supermultiplet on the nine-dimensional worldvolume of the D8-brane. Both problems, the gauge-invariance of the WZ term and the supersymmetry on the worldvolume, can be solved simultaneously by introducing a worldvolume gauge vector $b_{1}$ transforming with respect to the pull-back of the gauge parameter of the 2-form as $\delta b_{1}=-\Sigma_{1}$, which implies that $\mathcal{F}_{2} \equiv d b_{1}+B_{2}$ is gauge-invariant. A gauge-invariant WZ term is then given by ${ }^{3}$

$$
\mathcal{L}_{\mathrm{WZ}}=e^{\mathcal{F}_{2}} C .
$$

Here we use the standard notation where all RR potentials are contained in the formal sum $C \equiv C_{1}+C_{3}+C_{5}+\ldots$ To obtain the WZ term for the D8-brane one should project eq. (1.3) onto 9-forms. It is easily seen that this WZ term is invariant under the transformations $\delta C=d \lambda+H_{3} \lambda$ which generalizes eq. (1.2) to the other RR potentials, and using the Bianchi identity $d \mathcal{F}_{2}=H_{3}$. At the same time the introduction of a vector $b_{1}$ on the worldvolume, together with the transverse embedding scalar, fills a ninedimensional vector multiplet. We stress that when constructing this gaugeinvariant WZ term for the D8-brane one considers the transformation rules of the un-deformed IIA supergravity, which implies that the curvature $G_{10}$ of the RR 9-form $C_{9}$ is zero. This means that one is only considering here the D8-brane as a test brane thereby ignoring the back-reaction of the D8-brane on the supergravity background, ${ }^{4}$ which would indeed lead to the solution (1.1) of the Romans theory.

The existence of a gauge-invariant WZ term is a necessary but not sufficient requirement for the existence of the D8-brane. The full worldvolume action also contains kinetic terms for the worldvolume fields. This worldvolume action describes the dynamics of a single domain wall which does not constitute a finite-energy object by itself. For that one needs to introduce more domain walls and orientifolds as well. However, without going into the details of the precise construction, the requirement of a gauge-invariant WZ term consistent with supersymmetry on the worldvolume is a useful criterion which was applied in $[8,9,10,11]$ to determine all the supersymmetric branes that occur in IIA/IIB string theory compactified on a torus. In particular, this analysis shows that for the case of branes of codimen-

\footnotetext{
${ }^{3}$ The fact that $\mathcal{F}_{2}$ occurs non-polynomially in the WZ term is related to the fact that $\mathcal{F}_{2}$, like $B_{2}$, has scaling weight zero.

${ }^{4}$ One can also consider the D-branes as test branes in a deformed supergravity background, see, e.g., [7].
} 


\begin{tabular}{|c|c|c|}
\hline$D$ & $\mathrm{U}$ & $\mathrm{T}$ \\
\hline \hline IIA & $\mathbb{R}^{+}$ & 1 \\
\hline IIB & $S L(2, \mathbb{R})$ & 1 \\
\hline 9 & $S L(2, \mathbb{R}) \times \mathbb{R}^{+}$ & $S O(1,1)$ \\
\hline 8 & $S L(3, \mathbb{R}) \times S L(2, \mathbb{R})$ & $S L(2, \mathbb{R}) \times S L(2, \mathbb{R})$ \\
\hline 7 & $S L(5, \mathbb{R})$ & $S L(4, \mathbb{R})$ \\
\hline 6 & $S O(5,5)$ & $S O(4,4)$ \\
\hline 5 & $E_{6(6)}$ & $S O(5,5)$ \\
\hline 4 & $E_{7(7)}$ & $S O(6,6)$ \\
\hline 3 & $E_{8(8)}$ & $S O(7,7)$ \\
\hline
\end{tabular}

Table 2: This table indicates the continuous global U-duality and T-duality symmetries in dimensions $3 \leq D \leq 10$.

sion 2, 1 or 0 (that is, defect branes, domain walls and space-filling branes) the number of supersymmetric branes is lower than the dimension of the Uduality representation of the corresponding form fields. The same conclusion was reached by the analysis of [12], where the supersymmetric branes were counted as those corresponding to potentials associated to the real roots of the Kac-Moody algebra $E_{11}$ [13]. As far as domain walls are concerned, the outcome of this analysis, given in each dimension in terms of representations of the U-duality and T-duality groups (see Table 2 ) is summarized in Table $3[12,11]$. The U-duality representations of the $p$-form fields in dimensions $D \geq 3$ can be obtained either by using the tensor hierarchy formalism [14, 15], the Kac-Moody algebra $E_{11}[16,17]$, a Borcherds algebra approach $[18,19]$ or superspace methods [20].

This analysis, which gives the half-supersymmetric domain walls that can be introduced as probes in the undeformed supergravity theories in any dimensions, leaves unsolved the problem of determining which deformation of the supergravity theories these branes induce when the back-reaction with supergravity is taken into account. In the ten-dimensional case it was not difficult to guess which deformed IIA supergravity theory the D8 brane is 


\begin{tabular}{|c|c||c|c|c|c|c|c|}
\hline$D$ & U repr. & $\alpha=0$ & $\alpha=-1$ & $\alpha=-2$ & $\alpha=-3$ & $\alpha=-4$ & $\alpha=-5$ \\
\hline IIA & 1 & & 1 & & & & \\
\hline 9 & $2 \subset \mathbf{3}$ & & 1 & - & 1 & & \\
\hline 8 & $6 \subset(\mathbf{6}, \mathbf{2})$ & & $(\mathbf{1}, \mathbf{2})$ & - & $4 \subset(\mathbf{3}, \mathbf{2})$ & & \\
\hline 7 & $20 \subset \overline{\mathbf{4 0}}$ & & $\mathbf{4}$ & $4 \subset \mathbf{1 0}$ & $12 \subset \overline{\mathbf{2 0}}$ & & \\
\cline { 2 - 8 } & $5 \subset \overline{\mathbf{1 5}}$ & & & $4 \subset \overline{\mathbf{1 0}}$ & - & $\mathbf{1}$ & \\
\hline 6 & $80 \subset \mathbf{1 4 4}$ & & $\mathbf{8}_{\mathrm{S}}$ & $32 \subset \mathbf{5 6} \mathbf{C}_{\mathrm{y}}$ & $32 \subset \mathbf{5 6} \mathbf{S}_{\mathrm{y}}$ & $\mathbf{8}_{\mathrm{C}}$ & \\
\hline 5 & $216 \subset \mathbf{3 5 1}$ & & $\overline{\mathbf{1 6}}$ & $80 \subset \mathbf{1 2 0}$ & $80 \subset \mathbf{1 4 4}$ & $40 \subset \mathbf{4 5}$ & \\
\hline 4 & $576 \subset \mathbf{9 1 2}$ & & $\mathbf{3 2}$ & $160 \subset \mathbf{2 2 0}$ & $192 \subset \mathbf{3 5 2}$ & $160 \subset \mathbf{2 2 0}$ & $\mathbf{3 2}$ \\
\hline 3 & $2160 \subset \mathbf{3 8 7 5}$ & $\mathbf{1}$ & $\overline{\mathbf{6 4}}$ & $280 \subset \mathbf{3 6 4}$ & $448 \subset \mathbf{8 3 2}$ & $560 \subset \mathbf{1 0 0 1}$ & $448 \subset \overline{\mathbf{8 3 2}}$ \\
& & & & & & $14 \subset \mathbf{1 0 4}$ & \\
\hline$\leq-6$ & & & & & $280 \subset \mathbf{3 6 4}-6$ & $\mathbf{6 4}-7$ & $\mathbf{1}_{-8}$ \\
\hline
\end{tabular}

Table 3: The number of supersymmetric domain walls in different dimensions. The 7D case is discussed in Subsection 2.1.2. The representations at the right of the double vertical line indicate T-duality representations. The number $\alpha$ denotes the scaling of the mass $M$ of the brane with the string coupling $g_{s}$, i.e., $M \sim g_{s}^{-\alpha}$. In the last row, where the domain walls with $\alpha \leq-6$ occur, we have indicated the value of $\alpha$ with a sub-index.

a solution of, as there is only one deformation characterized by the single parameter $m$. In $D<10$ dimensions, all deformations of the maximal supergravity theories turn out to be gauged supergravities, and they are nicely classified in a U-duality covariant way by the so-called embedding tensor formalism [14]. The embedding tensor is an object belonging to a given representation of the U-duality group describing how the gauge group is embedded inside U-duality. Correspondingly, the theory admits $(D-1)$-form potentials belonging to representations which are dual to those of the embedding tensor, and whose $D$-form field strengths are related to the embedding tensor by duality $[16,17,15]$. However, since there are more $(D-1)$-form potentials than there are half-supersymmetric domain walls that allow supersymmetric WZ terms, not all gauged supergravities admit such half-supersymmetric 
domain-wall solutions. One of the purposes of this work is to find out which gauged supergravities correspond to half-supersymmetric domain walls allowing supersymmetric WZ terms.

A partial classification of half-supersymmetric domain-wall solutions of maximal supergravity theories was performed in [21] by considering all possible gauged theories in $D$ dimensions that arise as Scherk-Schwarz reductions from $D+1$ dimensions. Indeed, by considering a "vertical" dimensional reduction of a defect-brane solution which is magnetically charged under a given axion in $D+1$ dimensions, one obtains a domain-wall solution, but at the same time one has to impose for consistency that the axion depends linearly on the compactification coordinate, thus leading to a gauged theory in $D$ dimensions. All gaugings in $D \geq 7$ obtained with this method, and the corresponding domain wall solutions, were classified. We will comment at several places in the paper when our general analysis reproduces the results of $[21]$.

There is one subtlety in $D<10$ that does not occur in $D=10$ dimensions: it turns out that there are gauged supergravities that allow for half-supersymmetric domain-wall solutions that do not correspond to the half-supersymmetric branes following from the WZ term analysis. To distinguish between the different domain walls we will call the ones that do satisfy the WZ term criterion the elementary domain walls. The dynamics of the other domain walls cannot be described by a supersymmetric worldvolume action, and they are interpreted as bound states of the elementary domain walls. We have already seen in the case of the Romans theory that deforming a massless supergravity theory leads to a rearrangement of the degrees of freedom (see Table 1). In this work we will define the minimal gaugings as those for which this rearrangement is minimal, i.e., the minimal number of fields changes behaviour. Correspondingly the subgroup of the U-duality group that remains as a global symmetry of the deformed theory is maximal. We will see that all the gaugings that allow for elementary domain-wall solutions are minimal. We will also determine the gaugings that allow for domain-wall solutions describing threshold bound states, that is bound states preserving the same amount of supersymmetry as the elementary domain walls, as well as the gaugings that allow for domain-wall solutions describing non-threshold bound states, that have less supersymmetry.

The existence of threshold bound states shows that there are different elementary domain walls that satisfy the same BPS condition. This degeneracy does not occur for branes with three or more transverse directions. 
In general, the possible BPS conditions are in 1-1 correspondence with the central charges of the supersymmetry algebra. Therefore, for branes with three or more transverse directions one finds a 1-1 correspondence between these branes and the central charges [22]. This is not the case for branes with less than three transverse directions. For defect branes, i.e. branes with two transverse directions, one finds a double degeneracy: each BPS condition is satisfied by two defect branes which are related to each other by an S-duality transformation [23]. In this work we will spell out what the precise degeneracy structure is in the case of domain walls.

It turns out that several of the results derived in this work can be understood from the point of view of the $E_{11}$ Kac-Moody algebra [13]. This applies in particular to the classification of the half-supersymmetric domain walls, the analysis of the domain-wall solutions and the structure of the BPS conditions. We will at several places in the paper present this alternative $E_{11}$ point of view.

This work is organized as follows. In section 2 we classify the elementary supersymmetric domain walls by requiring the existence of a gauge-invariant WZ term that is consistent with supersymmetry on the worldvolume. We discuss an alternative derivation making use of the real roots of the $E_{11}$ Kac-Moody algebra. In section 3 we discuss which gauged supergravities correspond to these elementary supersymmetric domain walls. To illustrate our methods we present explicit results for the 9D, 8D and 7D gaugings. Next, in section 4 , we discuss the supersymmetric domain-wall solutions to these gauged supergravities. We point out that there exists a wider class of gauged supergravities that allow many more supersymmetric domain wall solutions. These solutions correspond to threshold and non-threshold bound states of the elementary domain walls discussed in the previous two sections. We will discuss the same solutions from an $E_{11}$ point of view. In section 5 we show that there is a relation between the number of elementary domain walls and the number of 2 -form $(4 \leq D \leq 10)$ and 1 -form (3D) central charges in the supersymmetry algebra after one takes into account the degeneracy of the BPS conditions involved. We discuss the relation between these BPS conditions and $E_{11}$. Finally, in section 6 we present our conclusions. 


\section{Classifying Supersymmetric Domain Walls}

In this section, we set the scene by reviewing how to classify supersymmetric domain walls by two different routes. The first is the analysis of supersymmetric WZ terms as a necessary condition for the existence of such domain walls $[8,9,10,11]$. The second approach uses properties of the corresponding roots of the Kac-Moody algebra $E_{11}[12]$. We phrase our discussion in a U-duality covariant way.

The form fields of the maximal supergravity theories in any dimension $D$, which include the propagating forms $A_{p}$, with $p \leq[D / 2]-1$, their magnetic duals $A_{D-p-2}$ together with the non-propagating forms $A_{D-1}$ and $A_{D},{ }^{5}$ were classified in $[16,17,15]$ in terms of their U-duality representations. The WZ-term analysis of $[8,9,10,11]$, and the $E_{11}$ analysis of [12] led to the conclusion that while for forms of rank less than $D-2$, that is for branes of codimension greater than 2, there are as many branes as the dimension of the U-duality representations of the corresponding fields, for branes of codimension 2, 1 and 0 the following results hold:

- When the representation is reducible, not all the corresponding irreducible representations are associated to branes. The branes always correspond to the highest-dimensional irreducible representation of the associated form, with the exception of the 5 -branes in $D=6, D=7$ and $D=8$, in which case the vector branes (that is the branes supporting a worldvolume vector multiplet) belong to the highest dimensional irreducible representation while the tensor branes (that is the branes supporting a worldvolume tensor multiplet) belong to the second highest-dimensional one.

- For a given irreducible representation, there are fewer supersymmetric branes than components of the representation. More precisely, the halfsupersymmetric branes belong in all cases to the highest-weight orbit of the corresponding representation $[12,11]$, but while the number of branes of codimension greater than 2 are as many as the dimension of the representation, for branes of codimension 2,1 and 0 the constraints that define the highest-weight orbit are stronger and one always gets fewer branes than the number of component of the corresponding representation.

\footnotetext{
${ }^{5}$ Although non-propagating, these fields can be introduced in the undeformed supersymmetry algebra $[6,24]$.
} 
In the remainder of this section, we will first review how to derive the number of half-supersymmetric branes from the analysis of the WZ term $[8$, $9,10,11]$. This will be done by deriving as an example the number of defect branes and domain walls in seven dimensions. We will then review how the same result can be obtained by counting the real roots of the $E_{11}$ algebra [12]. In Table 3 we have listed the number of domain walls resulting from this analysis. For completeness, we give the decomposition under T-duality of the U-duality representations and of the corresponding number of branes.

\subsection{Domain walls and supersymmetric WZ terms}

We consider maximal supergravity theories in $D \geq 3$ space-time dimensions, whose global symmetries $G=E_{11-D}$ are listed in the second column of Table 2. In order to write down a supersymmetric and gauge invariant WZ term for a $p$-brane of the $D$-dimensional theory one has to consider at leading order the pull-back to the worldvolume of the appropriate $(p+1)$-form potential. Given that such field transforms with respect to the gauge parameters of the lower-rank fields, this term alone cannot be gauge-invariant, and one has to add terms of the form $A \wedge \mathcal{F}$, where $\mathcal{F}$ are the field-strengths of suitably introduced worldvolume fields. The construction was reviewed in the Introduction for the case of D-branes in ten dimensions, where the gauge transformations of the RR fields force the introduction of a worldvolume vector, so that the resulting WZ term (1.3) is gauge-invariant. The necessary condition for the $p$-brane to be supersymmetric is then that the worldvolume fields (including the transverse scalars) that couple to this brane fill out the bosonic sector of a supermultiplet of the corresponding world-volume supersymmetry, that is either a vector (any $p$ ) or a tensor $(p=5)$ multiplet. In the case of the D-branes in 10 dimensions this is indeed the case as one always gets a vector plus $10-p-1$ transverse scalars, which is indeed a vector multiplet in $p+1$ dimensions.

We will now review how this works explicitly in $D=7$. In this case, the global symmetry group is $S L(5, \mathbb{R})$ and the form fields are $(M, N, P=$ $1, \ldots, 5)$ 


\begin{tabular}{|c|c|}
\hline$A_{1[M N]}$ & 1-form fields in the $\overline{\mathbf{1 0}}$ \\
\hline$A_{2}^{M}$ & 2 -form fields in the 5 \\
\hline$A_{3 M}$ & 3-form fields in the $\overline{\mathbf{5}}$ \\
\hline$A_{4}^{[M N]}$ & 4-form fields in the $\mathbf{1 0}$ \\
\hline$A_{5 M^{N}}$ & 5 -form fields in the (adjoint) 24 \\
\hline$N), A_{6}^{[M N], P}$ & 6 -form fields in the $\overline{\mathbf{1 5}} \oplus \overline{\mathbf{4 0}}$ \\
\hline
\end{tabular}

The 6 -form in the $\overline{\mathbf{4 0}}$ satisfies the irreducibility constraint $A_{6}^{[M N, P]}=0$. There are also 7 -form fields in the $\mathbf{5} \oplus \mathbf{4 5} \oplus \mathbf{7 0}$, but they will be of no importance for our discussion. We will only write down the leading WZ terms, that is the terms of the form $A+A \wedge \mathcal{F}$, and not terms which are higher order in $\mathcal{F}$ (although we know that such higher order terms are needed for gauge invariance). Besides, we will not determine the actual coefficient of each term: we will assume that if a given term can be written, it will actually occur in the WZ term with non-zero coefficient. We will now proceed with the analysis of the WZ terms of the defect branes and the domain walls in $D=7$.

\subsubsection{Example: $D=7$ defect branes}

We start by describing the WZ term analysis for 4-branes (defect branes), which are charged with respect to the 5 -form field $A_{5 M}{ }^{N}$. The WZ term is of the form

$$
\mathcal{L}_{W Z}^{p=4} \sim A_{5 M}{ }^{N}+A_{4}{ }^{N P} \mathcal{F}_{1 P M}+A_{3 M} \mathcal{F}_{2}{ }^{N}-\frac{1}{5} \delta_{M}^{N}\left(A_{4}{ }^{Q P} \mathcal{F}_{1 P Q}+A_{3 P} \mathcal{F}_{2}{ }^{P}\right) .
$$

We have not written out terms containing $\mathcal{F}_{3}$ and $\mathcal{F}_{4}$, because we assume that they are related to $\mathcal{F}_{2}$ and $\mathcal{F}_{1}$ by five-dimensional world-volume Hodge duality. ${ }^{6}$ The field strengths $\mathcal{F}_{p}$ are the field strengths of world-volume $(p-1)$-form fields augmented by $p$-form Stückelberg shifts of the pull-backs. More precisely

$$
\mathcal{F}_{1 P Q}=d a_{0 P Q}+A_{1 P Q}
$$

\footnotetext{
${ }^{6}$ We assume that the field-strengths $\mathcal{F}_{n}$ and $\mathcal{F}_{p+1-n}$ are related by worldvolume Hodge duality. We will make a similar assumption for the other WZ terms discussed in this section.
} 
with world-volume scalars $a_{0} P Q$ in the $\overline{\mathbf{1 0}}$ of $S L(5, \mathbb{R})$. Similarly, $\mathcal{F}_{2}^{M}$ is a gauge-invariant field-strength for the worldvolume field $a_{1}^{M}$. The last parenthesis in (2.1) is needed to ensure that the WZ term is in the traceless adjoint of $S L(5, \mathbb{R})$.

In order to determine which components of (the pull-back of) $A_{5} M^{N}$ couple to supersymmetric branes, we will now consider $A_{5} M^{N}$ for fixed $M$ and $N$. By analyzing eq. (2.1), one can see that in order to describe a single vector multiplet on the worldvolume (that is one vector and five scalars), one has to impose that $M \neq N$, so that the term in parenthesis is not present. Then, the term $A_{4}^{N P} \mathcal{F}_{1 P M}$ gives a scalar for each allowed $P$, which is three possibilities because $P$ is different from both $M$ and $N$. The term $A_{3 M} \mathcal{F}_{2}{ }^{N}$ gives one vector. Finally, there are two transverse scalars, making up a total of one vector and five scalars. This is the right field content for a vector supermultiplet on the five-dimensional world-volume and the necessary criterion for a supersymmetric 4-brane is fulfilled.

On the other hand, if $M=N$, the last parenthesis in (2.1) does not vanish and there are many more fields that couple to the world-volume theory. In fact, all 5 vector fields contribute as do all the 10 scalars; these cannot be grouped into world-volume supermultiplets and therefore the WZ term cannot be supersymmetrized. The case $M=N$ does not correspond to a supersymmetric brane. In all, only 20 out of the 24 components of $A_{5} M^{N}$ couple to supersymmetric branes. These components fill up the highestweight orbit in the 24 .

\subsubsection{Example: $D=7$ domain walls}

We now proceed to the case of interest here, namely domain walls in $D=$ 7. There are two distinct cases to consider since the 6-forms come in two different representations. The WZ term on the six-dimensional world-volume for the $\overline{\mathbf{1 5}}$ representation is

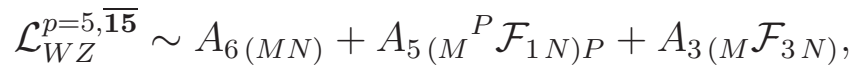

where $\mathcal{F}_{3 N}$ is the field-strength of a new world-volume 2-form field $a_{2 N}$. Following our worldvolume duality assumption, we must assume that this field-strength enjoys world-volume Hodge self-duality. Now the counting of world-volume fields works as follows. If $M=N$, there is a single self-dual tensor field from the last term, while the summation index $P$ has to be different from $M=N$ and there are therefore four scalar fields from the 
middle term. Together with the single transverse scalar this gives a selfdual tensor plus five scalars which is exactly the right content for a tensor multiplet on the six-dimensional world-volume. There are five choices for $M$ (equal to $N$ ) so that there are five supersymmetric tensor domain walls, again related to the highest-weight orbit.

If $M \neq N$, one obtains two tensor fields and seven scalar fields from (2.3). Together with the transverse scalar these do not form supermultiplets on the world-volume and therefore there is no supersymmetric domain wall in this case. In summary, only 5 out of the 15 6-forms couple to (elementary) supersymmetric tensor domain walls.

Turning to the 6 -forms in the $\overline{\mathbf{4 0}}$ of $S L(5, \mathbb{R})$, the WZ term looks like

$$
\begin{aligned}
\mathcal{L}_{W Z}^{p=5, \overline{40}} \sim & A_{6}^{M N, P}+A_{5 Q}{ }^{P} \mathcal{F}_{1 R S} \epsilon^{M N Q R S}+A_{4}^{M N} \mathcal{F}_{2}^{P} \\
& -\left(A_{5 Q}{ }^{[P} \mathcal{F}_{1 R S} \epsilon^{M N] Q R S}+A_{4}^{[M N} \mathcal{F}_{2}^{P]}\right)
\end{aligned}
$$

where the second line is needed to ensure that the irreducibility constraint of the $\overline{\mathbf{4 0}}$ is satisfied. When counting the world-volume fields one has to distinguish between the case $P=M$ (or equivalently $P=N$ ) and the case where all three indices are different. Starting with the former case when $P=$ $M$, the second line in (2.4) vanishes and the last term of the first line shows that there is a single vector field. Moreover, the antisymmetric summation indices $R$ and $S$ can take only three different values, so that there are three scalar fields. Together with the transverse scalar this gives four scalar fields. This is precisely the right number for a half-maximal vector multiplet in six world-volume dimensions. Counting the number of supersymmetric domain walls thus obtained, we find 20, ten from when $P=M$ and ten from when $P=N$ in the antisymmetric pair $[M N]$.

Performing the analysis in the case when all indices $M, N, P$ are different, one ends up with a field content that does not fit into supermultiplets. In total there are then 20 supersymmetric domain walls in the $\overline{\mathbf{4 0}}$, corresponding to the dimension of the highest-weight orbit.

A similar analysis can be performed in all dimensions. The result is given in Table 3. This table lists the U-duality and T-duality representations of all elementary supersymmetric domain walls that possess a supersymmetrizable WZ term. Note that only for $D=3,4,6$, where we have real representations, an elementary supersymmetric domain wall with given $\alpha$ transforms under 
S-duality into another domain wall with another value $\alpha^{\prime}$ given by:

$$
\alpha^{\prime}=-\alpha-4 \frac{D-1}{D-2} .
$$

For the other dimensions S-duality does not commute with T-duality and the transformations properties are more complicated.

\subsection{Supersymmetric domain walls and $E_{11}$}

The same classification of supersymmetric domain walls can be obtained independently from an analysis of the $E_{11}$ roots associated with the spacetime $p$-forms [12]. The infinite-dimensional Lorentzian Kac-Moody algebra $E_{11}$ reproduces nicely the tensor hierarchy of $p$-form fields that occurs in maximal supergravity $[16,17]$. In this language, one can obtain all the $p$ form fields in $D$ space-time dimensions and in a given representation of the U-duality group $E_{11-D}$ by decomposing the adjoint representation of $E_{11}$ under its $E_{11-D} \times G L(D, \mathbb{R})$ subalgebra. This decomposition produces an infinite number of fields but only a finite number of $p$-forms along with their U-duality representation, which are indeed the $p$-forms of the $D$-dimensional maximal supergravity theory.

Together with the above decomposition of the adjoint one also obtains root vectors $\alpha$ of the $E_{11}$ algebra that are associated with the various components of the $p$-forms. In order to decide which of these correspond to supersymmetric branes one has to recall that the inner product on the space of root vectors of $E_{11}$ is Lorentzian (whence the name Lorentzian Kac-Moody algebra). This means that root vectors can be either space-like (and are then called real roots), light-like (and are then called null roots) or time-like (and are then called purely imaginary). Often the last two cases are combined such that one is left with only the distinction between real $\left(\alpha^{2}>0\right)$ and imaginary roots $\left(\alpha^{2} \leq 0\right)$.

A given $p$-form transforms under U-duality such that in general the root vectors of the components can be either real or imaginary. The simple rule for classifying supersymmetric solutions is now that only those components that are associated with real roots correspond to supersymmetric branes whereas those components that are associated with imaginary roots are not supersymmetric. The solutions corresponding to real roots were discussed from a coset model point of view in $[25,26,27,28]$. That the solutions for imag- 
inary roots are not supersymmetric was explicitly checked in a particular representative case in [29].

The real roots relevant for domain walls can be easily generated and classified by using the language of orbits under the U-duality group. They are always in the orbit of the highest weight of a given U-duality representation of the $(D-1)$-forms (if the highest weight is a real root). This can be viewed alternatively as the Weyl group orbit of the highest weight, similar to the analysis in [30]. For example, in the $D=7$ case that was discussed above, the highest weight of the $\overline{\mathbf{1 5}}$ six-forms $A_{M N}$ is given by $A_{11}$ (by choosing an ordering of the five directions of the fundamental of $S L(5, \mathbb{R})$ and suppressing the space-time form index on $\left.A_{6, M N}\right)$. Its orbit corresponds to all components $A_{M M}$, i.e., those where the two indices are equal. These are the five real roots contained in the $\overline{\mathbf{1 5}}$ representation and we recover the counting and the same components coupling to supersymmetric tensor domain walls as we did from the analysis of the WZ term. For the six-forms in the $\overline{\mathbf{4 0}}$, the highest weight is given by the component $A^{12,1}$. The highest-weight orbit consists then of all components of $A^{M N, P}$ where $P=M$ or $P=N$. Therefore we arrive again at the same criterion and counting as from the analysis of the WZ term.

\section{Domain Walls and Gauged Supergravity}

In the previous section we reviewed the derivation of the elementary halfsupersymmetric domain walls in any maximal supergravity theory. These are all the domain walls, i.e. $(D-2)$-branes, of a $D$-dimensional theory that admit a half-supersymmetric effective action containing only one supersymmetric multiplet, that is either a vector (for any $D$ ) or a tensor multiplet (for $D=7)$. As we have seen in the introduction for the case of Romans IIA in ten dimensions, the presence of such a domain wall automatically induces a deformation of the supergravity theory, that is the supergravity theory is gauged. Maximal gauged supergravities in all dimensions have been classified in $[14,31]$ in terms of the so-called "embedding tensor", describing in a U-duality covariant way how the gauge group embeds inside the U-duality group. Denoting with $M_{1}$ the index of the U-duality representation to which the 1 -forms belong, and with $\alpha$ the adjoint representation, the gauging leads to covariant derivatives

$$
\partial_{\mu} \mathbb{I}-g A_{\mu, M_{1}} \Theta_{\alpha}^{M_{1}} t^{\alpha}
$$


where $\Theta_{\alpha}^{M_{1}}$ is the embedding tensor and $t^{\alpha}$ are the generators of the U-duality group. Consistency with maximal supersymmetry and gauge symmetry imposes constraints on the U-duality representations the embedding tensor belongs to, and it turns out that these representations are exactly conjugate to the representations of the $(D-1)$-forms in the theory $[16,17,15]$. As we have reviewed in the previous section, the elementary half-supersymmetric $(D-2)$-branes are fewer than the number of U-duality components of the corresponding $(D-1)$-form potentials, and in particular they correspond to the highest-weight orbit of the highest-dimensional irreducible representation (with the exception of the seven-dimensional case, where there are vector domain walls in the highest-weight orbit of the $\overline{\mathbf{4 0}}$ and tensor domain walls in the highest-weight orbit of the $\overline{\mathbf{1 5}}$ ). This means that these domain walls are associated to a particular class of gauged supergravities, corresponding to an embedding tensor having only non-zero components along these highestweight orbits. The aim of this section is to characterize these gauged theories.

As eq. (3.1) shows, the embedding tensor groups together a subset of the abelian vectors of the ungauged theory to form the adjoint of the gauge group, whose generators are

$$
X^{M_{1}}=\Theta_{\alpha}^{M_{1}} t^{\alpha}
$$

and whose commutation relations are given by

$$
\left.\left[X^{M_{1}}, X^{N_{1}}\right]=f^{M_{1} N_{1}}{ }_{P_{1}} X^{P_{1}}, \quad \text { with } \quad f^{M_{1} N_{1}}{ }_{P_{1}}=\left(X^{\left[M_{1}\right.}\right)_{P_{1}}{ }^{N_{1}}\right]
$$

where $\left(X^{M_{1}}\right)_{P_{1}}^{N_{1}}$ is given as in eq. (3.2) with the generators $t^{\alpha}$ acting on the representation of the 1 -forms. Consistency of the gauge algebra not only imposes constraints on the representation of the embedding tensor that we just mentioned (the so-called linear constraints) but also the quadratic constraints

$$
\Theta_{\gamma}^{P_{1}}\left(X^{M_{1}}\right)_{P_{1}}^{N_{1}}=\Theta_{\alpha}^{M_{1}} \Theta_{\beta}^{N_{1}} f_{\gamma}^{\alpha \beta}
$$

where $f^{\alpha \beta}$ are the structure constants of the U-duality group, i.e. $\left[t^{\alpha}, t^{\beta}\right]=$ $f^{\alpha \beta}{ }_{\gamma} t^{\gamma}$. Moreover, the 1-forms in general also have to transform under the gauge parameter of the 2 -forms $\Lambda_{1, M_{2}}$ as

$$
\delta_{\Lambda_{1}} A_{1, M_{1}}=-g Z^{M_{2}}{ }_{M_{1}} \Lambda_{1, M_{2}}
$$

where the constants $Z^{M_{2}} M_{1}$ satisfy the constraint

$$
Z^{M_{2}}{ }_{M_{1}} \Theta_{\alpha}^{M_{1}}=0
$$


and we denote with $M_{2}$ the representation to which the 2-forms belong. All these constraints guarantee that the gauging is consistent. This means that for instance while a subset of the abelian 1-forms of the ungauged theory form the adjoint of the gauge group, the remaining 1-forms can either be uncharged with respect to this gauge group or they are gauged away to give a mass to some of the 2 -forms in the theory. This gives rise to a hierarchy of forms that continues all the way to the space-filling $D$-forms.

As a prototypical, although somewhat degenerate example, one can consider the Romans mass deformation of the IIA theory. In this case the embedding tensor vanishes, while $Z$ corresponds to the Romans mass $m$. This means that the 1-form present in the massless theory is gauged away to give mass to the 2 -form, see Table 1 . In the rest of this section we will see how the rearrangement of the degrees of freedom works explicitly in the $D=9$, $D=8$ and $D=7$ gaugings. In each case we will select among all the possible gaugings the ones that correspond to the highest-weight orbit - that is the gaugings that admit elementary domain-wall solutions. We will see how each orbit of gaugings corresponds to a different rearrangement of the degrees of freedom, and eventually we will point out what are the basic features of the highest-weight orbit gaugings and how the degrees of freedom are rearranged in these particular cases. Here we anticipate the result, that is the highest-weight orbit gaugings are the deformations that lead to the minimal rearrangement of the degrees of freedom. Hence we call these gaugings minimal.

\subsection{The nine-dimensional gaugings}

In nine dimensions the global symmetry is $G L(2, \mathbb{R})$, and the 1-forms are $A_{1}, A_{1, a}$ in the $\mathbf{1} \oplus \mathbf{2}$. There is also a doublet of 2 -forms $A_{2, a}$ and a singlet 3 -form $A_{3}$. The gaugings of this theory have been classified in [32] and then reconsidered using the embedding tensor formalism in [33]. The linear constraints imply that the embedding tensor is $\Theta^{a}, \Theta^{a b}$ belonging to the $\mathbf{2} \oplus \mathbf{3}$, while the quadratic constraints are

$$
\Theta^{a} \Theta^{b c} \epsilon_{a b}=0 \quad \Theta^{(a} \Theta^{b c)}=0 \quad,
$$

which imply that the two embedding tensors cannot be turned on together.

There is a single orbit of gauged theories associated to $\Theta^{a}$. This corresponds to an $\mathbb{R}^{+}$gauging. The 1 -form $A_{1}$ is gauged away by a shift $\Theta^{a} \Lambda_{1, a}$, 
where $\Lambda_{1, a}$ is the parameter of the 2-form. If one takes $\Theta^{1}=1, \Theta^{2}=0$, then $A_{2,1}$ is massive, while $A_{2,2}$ is gauged away by the shift $\epsilon_{a b} \Theta^{b} \Lambda_{2}$, where $\Lambda_{2}$ is the gauge parameter of the 3 -form. Correspondingly, the 3 -form becomes massive.

Considering the $\Theta^{a b}$ gaugings, one has that the 1-forms $A_{1, a}$ have the shift gauge symmetry $\epsilon_{a b} \Theta^{b c} \Lambda_{1, c}$. There are three different orbits. Indeed, up to $S L(2, \mathbb{R})$ transformations, $\Theta^{a b}$ can be written as $\operatorname{diag}(1,1), \operatorname{diag}(1,-1)$ or $\operatorname{diag}(1,0)$. The first two cases, corresponding to an $S O(2)$ and an $S O(1,1)$ gauging respectively, have the property that both 1 -forms $A_{1, a}$ are gauged away, leading to two massive 2-forms, while in the third case only the 1 -form $A_{1,2}$ is gauged away by the parameter $\Lambda_{1,1}$. Correspondingly, only the 2 -form $A_{2,1}$ is massive and the other one remains massless. This last gauging, which is minimal because it gives the least amount of rearrangements of the degrees of freedom, is exactly the highest-weight orbit gauging corresponding to the two elementary half-supersymmetric domain walls that we discussed in the previous section. The analysis of the degrees of freedom for the $\Theta^{a b}$ gaugings is summarized in Table 4.

\begin{tabular}{|c||c|c|c|c|c|c|}
\hline gauging & $A_{1}$ & $A_{1,1}$ & $A_{1,2}$ & $A_{2,1}$ & $A_{2,2}$ & $A_{3}$ \\
\hline$\Theta^{11}=1 \Theta^{22}= \pm 1$ & massive & gauged & gauged & massive & massive & massless \\
\hline$\Theta^{11}=1 \Theta^{22}=0$ & massive & massless & gauged & massive & massless & massless \\
\hline
\end{tabular}

Table 4: The $\Theta^{a b}$ gaugings in nine dimensions. The last row corresponds to the minimal gauging leading to the elementary domain-wall solution.

\subsection{The eight-dimensional gaugings}

In eight dimensions the symmetry is $S L(3, \mathbb{R}) \times S L(2, \mathbb{R})$, and the propagating forms are the 1 -forms $A_{1, M a}$ in the $(\overline{\mathbf{3}}, \mathbf{2})$, the 2 -forms $A_{2}^{M}$ in the $(\mathbf{3}, \mathbf{1})$ and the 3 -forms $A_{1, a}$ in the $(\mathbf{1}, \mathbf{2})$ which satisfy a self-duality condition. The most general gaugings of this theory have been derived in $[34,35]$. The linear constraints select the embedding tensors $\Theta_{M N}{ }^{a}$ in the $(\overline{\mathbf{6}}, \mathbf{2})$ and $\Theta^{M a}$ in the 
$(\mathbf{3}, \mathbf{2})$, with quadratic constraints

$$
\begin{aligned}
& \epsilon_{a b} \Theta^{M a} \Theta^{N b}=0 \\
& \Theta_{M N}{ }^{(a} \Theta^{N b)}=0 \\
& \epsilon_{a b}\left(\epsilon^{M Q R} \Theta_{Q N}{ }^{a} \Theta_{R P}{ }^{b}+\Theta_{N P}{ }^{a} \Theta^{M b}\right)=0 .
\end{aligned}
$$

We know (see Table 3) that the elementary domain walls are associated to gaugings in the $(\overline{\mathbf{6}}, \mathbf{2})$. More precisely we know that there are 6 half-supersymmetric elementary domain walls corresponding to the following gaugings

$$
\begin{array}{ll}
\Theta_{11}{ }^{1} & \Theta_{11}{ }^{2} \\
\Theta_{22}{ }^{1} & \Theta_{22}{ }^{2} \\
\Theta_{33}{ }^{1} & \Theta_{33}{ }^{2}
\end{array}
$$

It is easy to see that each embedding tensor in eq. (3.9) satisfies the quadratic constraint (3.8).

\begin{tabular}{|c||c|c|c|c|c|c|c|}
\hline gauging & $A_{1,11}$ & $A_{1,12}$ & $A_{1, i 1}$ & $A_{1, i 2}$ & $A_{2}^{1}$ & $A_{2}^{i}$ & $A_{3, a}$ \\
\hline$\Theta_{11}{ }^{1}=1$ & massless & gauged & massive & massless & massive & massless & massless \\
\hline
\end{tabular}

Table 5: The minimal gauging in eight dimensions. The index $i=2,3$ labels a global $S L(2, \mathbb{R})$ symmetry inside the original $S L(3, \mathbb{R})$ which is preserved by the gauging.

All the gaugings in the $(\overline{\mathbf{6}}, \mathbf{2})$ can be obtained reducing the 11-dimensional theory over group manifolds [36]. For such gaugings one can show that imposing the quadratic constraints, and up to U-duality transformations, one can always consider the index $a$ to be in the 1 direction, and $\Theta_{M N}^{1}$ in the diagonal form

$$
\Theta_{M N}{ }^{1}=\operatorname{diag}\left(\mathbb{I}_{p},-\mathbb{I}_{q}, \mathbb{O}_{r}\right) \quad p+q+r=3
$$

leading to the gauge group $[36,37]$

$$
C S O(p, q, r)
$$


The structure constants of the gauge group are given by ${ }^{7}$

$$
f^{M N}{ }_{P}=\epsilon^{M N Q} \Theta_{P Q},
$$

leading to the following algebra

$$
\left[X^{1}, X^{2}\right]=\Theta_{33} X^{3}, \quad\left[X^{2}, X^{3}\right]=\Theta_{11} X^{1}, \quad\left[X^{3}, X^{1}\right]=\Theta_{22} X^{2}
$$

For $p=3, q=r=0$ one gets the $S O(3)$ gauging of [38], and one can obtain all the non-compact gaugings by group contraction and/or analytic continuation from $S O(3)$. The case $p=2, q=1, r=0$ is the $S O(2,1)$ gauging, while for $r=1$ one can have $p=2, q=0$, which is an $\operatorname{ISO}(2)$ gauging, or $p=1, q=1$, which is an $I S O(1,1)$ gauging. The minimal gaugings, associated to the elementary domain walls, have $p=1, q=0$, $r=2$, corresponding to a gauge group $C S O(1,0,2)$. Taking for instance $\Theta_{11}=1$ and $\Theta_{22}=\Theta_{33}=0$, this is the three-dimensional Heisenberg algebra

$$
\left[X^{2}, X^{3}\right]=X^{1} \quad, \quad\left[X^{1}, X^{2}\right]=\left[X^{1}, X^{3}\right]=0 \quad .
$$

The gauge fields which acquire a mass by the Higgs mechanism are $A_{1, i 1}$, while the shift symmetry $\Theta_{M N}^{b} \epsilon_{a b} \Lambda_{1}^{N}$ of $A_{1, M a}$ gauges away $A_{1,12}$ giving a mass to the 2 -from $A_{2}^{1}$. All the other gauge fields remain massless. In table 5 we have summarized this rearrangement. As in nine dimensions, this rearrangement of the degrees of freedom with respect to the ungauged theory is minimal and leads to the elementary domain walls.

\subsection{The seven-dimensional gaugings}

The ungauged seven-dimensional maximal supergravity theory has global U-duality symmetry $S L(5, \mathbb{R})$, and its gaugings are determined by the embedding tensors $\Theta_{M N, P}$ in the $\mathbf{4 0}$ and $\Theta^{M N}$ in the 15. The propagating forms are the 1 -forms $A_{1, M N}$ in the $\overline{\mathbf{1 0}}$ and the 2 -forms $A_{2}^{M}$ in the $\mathbf{5}$.

The elementary vector domain walls are associated to gaugings in the highest-weight orbit of the 40. These correspond to an embedding tensor of the form $\Theta_{M N, M}$ for fixed $M$ and $N$, giving in total 20 different gaugings. In general, for any embedding tensor of the form $\Theta_{M N, P}=v_{[M} w_{N] P}$, with $v_{M}$ a reference vector and with $w_{M N}$ symmetric, all the possible gaugings have been classified in [31], where it was shown that imposing the quadratic

\footnotetext{
${ }^{7}$ We have dropped here the $S L(2, \mathbb{R})$ index $a=1$ on $\Theta_{11}{ }^{1}$ for ease of notation.
} 


\begin{tabular}{|c||c|c|c|c|c|c|c|}
\hline gauging & $A_{1, i j}$ & $A_{1,12}$ & $A_{1,1 i}$ & $A_{1,2 i}$ & $A_{2}^{1}$ & $A_{2}^{2}$ & $A_{2}^{i}$ \\
\hline$\Theta_{12,1}=1$ & massive & gauged & massless & massless & massive & massless & massless \\
\hline
\end{tabular}

Table 6: The minimal gauging in the $\mathbf{4 0}$ in seven dimensions. The index $i=3,4,5$ labels a global $S L(3, \mathbb{R})$ symmetry inside the original $S L(5, \mathbb{R})$ which is preserved by the gauging.

constraints, and up to U-duality transformations, one can always consider $w_{M N}$ in the diagonal form ${ }^{8}$

$$
w_{M N}=\operatorname{diag}\left(\mathbb{I}_{p},-\mathbb{I}_{q}, \mathbb{O}_{r}\right) \quad p+q+r=4
$$

leading to the gauge group $C S O(p, q, r)$. The particular case of the highestweight orbit corresponds to the case $p=1, q=0, r=3$ leading to the minimal gauging $C S O(1,0,3)$. In this case we can consider as a representative of the highest-weight orbit the component $\Theta_{12,1}$. This leads to the gauge algebra [39]

$$
\left[X^{i}, X^{j}\right]=X^{i j} \quad i, j=3,4,5,
$$

where the indices $i, j=3,4,5$ label the $S L(3, \mathbb{R})$ which remains as a global symmetry of the deformed theory. The gauge fields, acquiring a mass by eating three of the axions, are $A_{1, i j}$. The shift symmetry of the 1-forms $\Theta_{M N, P} \Lambda_{1}^{P}$ gauges away $A_{1,12}$. The vectors $A_{1,1 i}$ and $A_{1,2 i}$ remain massless. The resulting rearrangement of the degrees of freedom is summarized in Table 6. One can compare this table with Table 3 of [21], where a particular example of this orbit of gaugings was obtained as a Scherk-Schwarz reduction from eight dimensions. We stress that among all possible gaugings in the $\mathbf{4 0}$, the highest weight one is the one that preserves the highest amount of global symmetries.

We now consider the gaugings associated to the elementary tensor domain walls. These gaugings belong to the highest-weight orbit in the $\mathbf{1 5}$, corresponding to an embedding tensor $\Theta^{M N}$ of the form $\Theta^{M M}$ for fixed $M$. In general, all possible gaugings in the $\mathbf{1 5}$ have been classified in [31], where

\footnotetext{
4.

${ }^{8}$ Note that, given a fixed reference vector, the indices of $w_{M N}$ effectively run from 1 to
} 
it was shown that imposing the quadratic constraints, and up to U-duality transformations, one can always consider $\Theta^{M N}$ in the diagonal form

$$
\Theta^{M N}=\operatorname{diag}\left(\mathbb{I}_{p},-\mathbb{I}_{q}, \mathbb{O}_{r}\right) \quad p+q+r=5,
$$

which again results in the gauge group $C S O(p, q, r)$. The minimal gaugings correspond to $p=1, q=0, r=4$, leading to the gauge group $C S O(1,0,4)$. Considering for instance $\Theta^{11}$ as the only non-vanishing component, one can see that the gauge vectors, acquiring a mass by the Higgs mechanism gauging away four axions, are $A_{1,1 i}$, with the index $i$ now labeling the directions $2,3,4,5$ in the fundamental of $S L(5, \mathbb{R})$. These indices label the global $S L(4, \mathbb{R})$ symmetry which is preserved by this gauging. As in the previous case, this is the highest amount of global symmetries that is preserved by any possible gauging in the $\mathbf{1 5}$. The other six vectors $A_{1, i j}$ remain massless, while one of the 2-forms is gauged away by the shift symmetry $\Theta^{M N} \Lambda_{2, N}$, where $\Lambda_{2, M}$ are the gauge parameters of the 3 -forms $A_{3, M}$ that in the ungauged theory are dual to the 2 -forms. Correspondingly, one of these 3 -forms acquires a mass. This is consistent with the counting of the degrees of freedom because in the gauged theory this 3-form satisfies a massive self-duality condition. The list of all the degrees of freedom for this gauging is summarized in Table 7. Again, one can compare this table with Table 4 of [21].

\begin{tabular}{|c||c|c|c|c|c|}
\hline gauging & $A_{1,1 i}$ & $A_{1, i j}$ & $A_{2}^{1}$ & $A_{2}^{i}$ & $A_{3,1}$ \\
\hline$\Theta^{11}=1$ & massive & massless & gauged & massless & massive \\
\hline
\end{tabular}

Table 7: The minimal gauging in the $\mathbf{1 5}$ in seven dimensions. The index $i=2,3,4,5$ labels a global $S L(4, \mathbb{R})$ symmetry inside the original $S L(5, \mathbb{R})$ which is preserved by the gauging.

In lower dimensions, all these results continue to hold, namely:

- the elementary domain walls are solutions of the gauged supergravity theories obtained by taking the embedding tensor to take values in the highest-weight orbit of the relevant representation;

- an embedding tensor taking values in the highest-weight orbit satisfies the quadratic constraints and leads to a minimal gauging; 
- this gauging preserves the highest amount of global symmetries among all possible gaugings in the same representation.

In the next section we will discuss domain-wall solutions, and show that one can obtain (non-elementary) half-supersymmetric domain-wall solutions also for gaugings that are not in the highest-weight orbit.

\section{Domain-wall Solutions}

In the previous two sections we have classified the elementary supersymmetric domain walls of toroidally compactified IIA/IIB string theory and specified the minimal gauged supergravity theories they correspond to. In this section we wish to investigate the domain-wall solutions of generic maximally supersymmetric gauged supergravities, including the non-minimal ones. We already discussed the 10D case in the introduction, in which case there is only one elementary D8-brane which is a solution of massive IIA supergravity. In the next two subsections we will discuss the situation both from a supergravity as well as from an $E_{11}$ point of view.

\subsection{Domain-wall solutions and supergravity}

Below we will discuss domain-wall solutions of gauged supergravity theories in $9 \mathrm{D}, 8 \mathrm{D}$ and $7 \mathrm{D}$, respectively.

$D=9$ : In 9D there are two distinct embedding tensors. One is a doublet $\Theta^{a}(a=1,2)$ and the other is a triplet $\Theta^{i}(i=1,2,3)^{9}$ of the U-duality group $G L(2, \mathbb{R})$. The projection operator for a domain wall is given by

$$
\frac{1}{2}\left(1 \pm \gamma_{y}\right) \epsilon_{0}=0
$$

where $\epsilon_{0}$ is a constant spinor and $y$ denotes the transverse direction. From an investigation of the Killing spinor equations it follows that the gauged supergravities corresponding to the $\Theta^{a}$ gaugings do not have any half-supersymmetric domain-wall solutions [32]. This in accordance with the fact that

\footnotetext{
${ }^{9}$ In the previous section we have denoted this embedding tensor as $\Theta^{a b}$, symmetric in $a b$. The relation between this object and $\Theta^{i}$ is $\Theta^{a b}=\Theta^{i} t_{i}^{a b}$, where $t_{i}^{a b}$ are the $S L(2, \mathbb{R})$ generators. This leads to the identifications $\Theta^{11}=\Theta^{+}, \Theta^{22}=\Theta^{-}$and $\Theta^{12}=\Theta^{3}$ (see eq. $(4.2))$.
} 
only the highest-dimensional $(D-1)$-form potentials couple to supersymmetric domain walls.

The $\Theta^{i}$ gaugings do allow half-supersymmetric domain-wall solutions which have been extensively investigated in [40]. Choosing a lightcone direction

$$
\Theta^{i}=\left(\Theta^{+}, \Theta^{-}, \Theta^{3}\right)=(1,0,0)
$$

one obtains a minimal $\mathbb{R}^{+}$-gauging (see the previous section). This minimal gauging allows an elementary domain-wall solution which can be oxidized to the IIB D7-brane solution. Choosing the other lightcone direction, i.e. $\Theta^{i}=$ $(0,1,0)$, a similar domain-wall solution and potential is obtained. This one oxidizes to the S-dual of the IIB D7-brane solution.

Choosing a non-light-cone direction, i.e.

$$
\Theta^{i}=(0,0,1)
$$

one obtains a non-minimal $S O(1,1)$-gauging. The corresponding gauged theory admits a supersymmetric domain wall that is not an elementary brane. Instead, this solution describes the threshold bound state of two elementary domain walls.

$D=8$ : As we have reviewed in the previous section, in $8 \mathrm{D}$ we have two embedding tensors. One embedding tensor $\Theta_{M N}{ }^{a}(M=1,2,3 ; a=1,2)$ transforms in the $(\overline{\mathbf{6}}, \mathbf{2})$ of the U-duality group $S L(3, \mathbb{R}) \times S L(2, \mathbb{R})$. The other embedding tensor $\Theta^{M a}$ transforms in the $(\mathbf{3}, \mathbf{2})$ representation. The supersymmetric domain walls of $8 \mathrm{D}$ maximal gauged supergravity have been extensively discussed in $[36,37]$. As expected, one finds that the lowest dimensional $(\mathbf{3}, \mathbf{2})$ representation does not lead to any supersymmetric domain-wall solutions. The highest-dimensional $(\overline{\mathbf{6}}, \mathbf{2})$ representation leads to 6 half-supersymmetric domain-wall solutions. The minimal gaugings corresponding to these elementary domain walls have been discussed in subsection 3.2.

The elementary domain walls can be obtained as truncations of the general domain-wall solutions given in $[36,37]$ which contain three independent harmonic functions. The results of $[36,37]$ show that there are many more supersymmetric domain-wall solutions corresponding to more general gaugings. For instance, the $I S O(2)$-gaugings or $S O(3)$-gaugings

$$
I S O(2): \Theta_{11}{ }^{1}=\Theta_{22}{ }^{1}=1 ; \quad S O(3): \Theta_{11}{ }^{1}=\Theta_{22}{ }^{1}=\Theta_{33}{ }^{1}=1
$$

allow supersymmetric domain walls with two and three independent harmonic functions, respectively. These supersymmetric domain-wall solutions 
do not correspond to elementary domain walls. Instead, they should be viewed as threshold bound states of the elementary domain walls. The 11D origin of these threshold bound states has been discussed in [37].

$D=7$ : In $7 \mathrm{D}$ there are two embedding tensors. One embedding tensor $\Theta_{M N, P}$ transforms in the $\mathbf{4 0}$ of the $S L(5, \mathbb{R})$ U-duality symmetry. The other embedding tensor $\Theta^{M N}$ transforms in the $\mathbf{1 5}$ representation. What is special in 7D is that domain walls are 5-branes and there are two types of them: domain walls with worldvolume vector multiplets and domain walls with worldvolume tensor multiplets. The $\Theta_{M N, P}$ lead to gaugings that allow vector domain walls, while the lower-dimensional embedding tensor $\Theta^{M N}$ also allows supersymmetric domain-wall solutions that have tensor instead of vector multiplets on the worldvolume.

A systematic investigation of the supersymmetric domain-wall solutions of 7D maximal gauged supergravity has not been performed so far. Following our general analysis, we expect that the minimal gaugings discussed in subsection 3.3 lead to 20 elementary vector domain-wall solutions and 5 tensor domain-wall solutions. It is interesting to consider the $\Theta^{M N}$ and $\Theta_{M N, P}$ gaugings together. Defining

$$
s=\operatorname{rank} \Theta_{M N, P} \quad t=\operatorname{rank} \Theta^{M N}
$$

where $\Theta_{M N, P}$ is understood as a rectangular $10 \times 5$ matrix, one finds the following re-arrangement of the 100 degrees of freedom carried by the different 1-forms, 2-forms and 3-forms [31], see Table 8. ${ }^{10}$ For $(s, t)=(1,0)$ and $(s, t)=(0,1)$ this table reproduces the results of Tables 6 and 7 , respectively. Note that the quadratic constraint ensures that $s+t \leq 5$.

\begin{tabular}{|c||c|c|c|c|}
\hline form & 1-forms & 2-forms & 2-forms & s.d. 3-forms \\
\hline mass & massless/massive & massless & massive & massive \\
\hline$\#$ & $10-s$ & $5-s-t$ & $s$ & $t$ \\
\hline
\end{tabular}

Table 8: The minimal gauging in the $\mathbf{4 0} \oplus \mathbf{1 5}$ in seven dimensions.

\footnotetext{
${ }^{10}$ We do not indicate how many of the 1-forms are massless or massive. This would require a more detailed analysis of the gauge transformations including the axions.
} 
Unlike the previous cases, since we are now dealing with two different BPS conditions (see also section 5), we expect this to lead to domain walls with less supersymmetry, i.e. $1 / 4$ supersymmetric domain walls. Indeed such 1/4-supersymmetric domain wall solutions are discussed in [21], where it is also shown that a particular representative of these gauged supergravity theories is obtained as a generalized Scherk-Schwarz reduction where two different axions acquire a linear dependence on the compactified coordinate. By reduction we expect similar 1/4-supersymmetric domain-wall solutions to occur in $D<7$ dimensions.

\subsection{Domain-wall solutions and $E_{11}$}

We now explain some of the algebraic mechanics of domain-wall solutions from a one-dimensional $E_{11}$ coset model point of view. A one-dimensional coset model based on $E_{11}$ with the so-called temporal involution was presented in [25] based on [41]. The coset model is given by a map

$$
\mathcal{V}: \mathbb{R} \rightarrow E_{11} / K^{*}\left(E_{11}\right), \quad \xi \mapsto \mathcal{V}(\xi),
$$

where $K^{*}\left(E_{11}\right)$ is the subgroup of $E_{11}$ fixed by the temporal involution [25]. This involution is such that $K^{*}\left(E_{11}\right)$ contains the Lorentz group $S O(10,1)$ rather than the compact rotation group $S O(11)$ that one would obtain with the standard Chevalley involution on $E_{11}{ }^{11}$ The action for the coset model is the standard one for null geodesic motion [41, 25, 44]

$$
S=\int d \xi \frac{1}{2 n}\langle\mathcal{P} \mid \mathcal{P}\rangle,
$$

with the Killing bilinear form $\langle\cdot \mid \cdot\rangle$ and where the lapse $n(\xi)$ is needed for reparametrization invariance of the geodesic and

$$
\mathcal{P}=\frac{1}{2}\left(\partial_{\xi} \mathcal{V} \mathcal{V}^{-1}+\left(\partial_{\xi} \mathcal{V} \mathcal{V}^{-1}\right)^{\#}\right),
$$

where (.)\# denotes the application of minus the temporal involution. Thinking of $\mathcal{V}$ as a matrix, the operation $\mathcal{V}^{\#}$ can be though of as transposing $\mathcal{V}$ and multiplying by the (generalization) of the Lorentz metric from left and right. $\mathcal{P}$ is then (roughly) the symmetric part of the Maurer-Cartan form.

\footnotetext{
${ }^{11}$ Using the temporal involution also groups together possible different choices of spacetime signature $[42,43]$.
} 
We note that the domain walls are the perfect playground for the onedimensional sigma model since domain walls depend only on one transverse direction which can be identified with the geodesic parameter $\xi$. This can be used to explicitly determine the space-time solution from a solution of the one-dimensional sigma model once a map between the coset variables and space-time variables is known [25, 45].

For our elementary branes we are interested in configurations that use only a single $E_{11}$ step operator $E_{\alpha}$ (for a given root $\alpha$ ) out of the infinitely many $[25,27,28,45]$, see also $[52,53]$. We parametrize the coset element then as

$$
\mathcal{V}=e^{\phi h_{\alpha}} e^{A E_{\alpha}}
$$

where $E_{\alpha}$ is the single step operator and $h_{\alpha}$ is its associated element in the Cartan subalgebra. We assume $E_{\alpha}+\left(E_{\alpha}\right)^{\#}$ to be associated with a compact direction. The reason for this that we are interested in brane solutions rather than cosmological solutions $[48,45]$. In this $S L(2, \mathbb{R})$ subsector (for real $\alpha$ ) all coset equations of motion are solved by $[48,45]$

$$
e^{2 \phi(\xi)}=H(\xi) \quad \text { and } \quad A= \pm H^{-1}
$$

for any harmonic function $H(\xi)$. The variable $A$ is cyclic in the coset dynamics and generates an effective potential for the scalar field $\phi$ similar to the scalar potential of the associated gauged supergravity. For imaginary roots $\alpha$ the solution looks very different [45].

Using the known standard dictionary for supergravity solutions [41], one expects the scalar field to be related to a combination of the dilaton fields in maximal supergravity in $D$ dimensions. Since the metric is also related to exponentials of $\phi$, a constant $\phi$ means in fact a flat space solution which is clearly not a domain wall. Since the dictionary is not established for imaginary roots the exact general space-time interpretation of the solution to the geodesic model is not known in that case. In the null case a thorough investigation was carried out in [29], showing that the corresponding space-time solution is not supersymmetric.

Example 1: The D8-brane of massive type IIA can be described in this language $[27,49]$. For this one requires the root vector $\alpha$ that is related to the 9 -form and the relation of $\alpha$ to a basis of metric components and dilaton. The detailed change of basis can be found for example in $[27,50,49,51]$. 


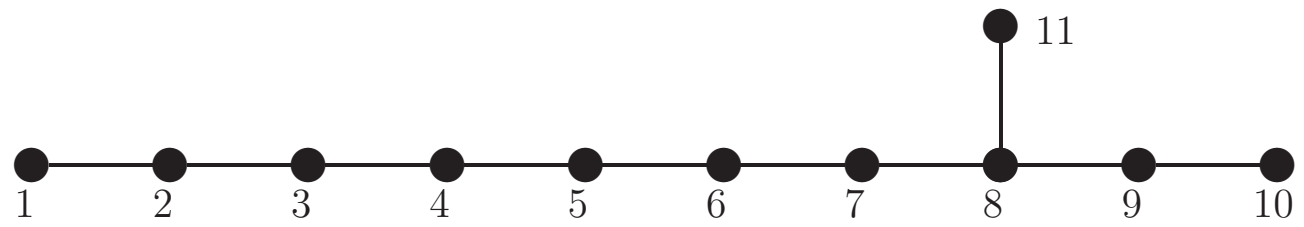

Figure 1: Dynkin diagram of $E_{11}$ with labelling of nodes.

Here, we only give the result. The root vector $\alpha$ for a D8-brane (in directions $1, \ldots, 9)$ is

$$
\alpha=\left(\frac{9}{16}, \frac{1}{16}, \frac{1}{16}, \frac{1}{16}, \frac{1}{16}, \frac{1}{16}, \frac{1}{16}, \frac{1}{16}, \frac{1}{16}, \frac{1}{16} ; \frac{5}{4}\right)=\left(p_{i} ; p_{\phi}\right) .
$$

From this root vector one obtains the diagonal metric and dilaton by taking $g_{i i}=\epsilon_{i} H^{2 p_{i}}$ and $e^{\phi}=H^{p_{\phi}}$, where $\epsilon_{i}= \pm 1$ is the signature of the $i$ th direction. This reproduces the Einstein frame metric (1.1). The form field from (4.10) also is correct compared to (1.1).

Example 2: As another example, we consider the intersection of two 1/2-BPS domain walls in $D=7$. As there are now several dilatonic scalars involved, we refrain from giving the full metric but only indicate the $E_{11}$ roots. Expanded on a basis of simple roots labeled according to the $E_{11}$ Dynkin diagram of figure 1 one can choose a 1/2-BPS vector domain wall with corresponding real root $\alpha_{\mathrm{v}}$ and a $1 / 2$-BPS tensor domain wall with corresponding root $\alpha_{\mathrm{t}}$ where

$$
\begin{aligned}
& \alpha_{\mathrm{v}}=\alpha_{2}+2 \alpha_{3}+3 \alpha_{4}+4 \alpha_{5}+5 \alpha_{6}+6 \alpha_{7}+6 \alpha_{8}+3 \alpha_{9}+\alpha_{10}+3 \alpha_{11}, \\
& \alpha_{\mathrm{t}}=\alpha_{2}+2 \alpha_{3}+3 \alpha_{4}+4 \alpha_{5}+5 \alpha_{6}+6 \alpha_{7}+6 \alpha_{8}+4 \alpha_{9}+2 \alpha_{10}+2 \alpha_{11} .
\end{aligned}
$$

One can check that these roots are Cartan orthogonal, i.e., they satisfy $\alpha_{\mathrm{v}}$. $\alpha_{\mathrm{t}}=0$ with respect to the Cartan inner product. They therefore correspond to an orthogonal intersection of $1 / 2$-BPS branes [26]. That the intersecting solution is 1/4-BPS can be inferred from the analysis of the BPS conditions of the following section. We remark that, from an M-theory perspective, the configuration above corresponds to the intersection of a KK7-monopole with an M5-brane. Bound states viewed from an algebraic perspective were also discussed in $[52,53]$. 


\section{Domain Walls and Central Charges}

In this section, we study the BPS conditions satisfied by elementary supersymmetric domain walls. We will do this first from a field theory perspective and, next, from an $E_{11}$ point of view.

\subsection{One central charge, many domain walls}

It is well-known that there is a 1-1 relation between the half-supersymmetric branes of maximal supergravity with more than or equal to three transverse directions and the central charges in the supersymmetry algebras with 32 supercharges $[22,54]$. It is less obvious what the precise relation is for the branes with less than three transverse directions. This is due to the fact that these branes are not asymptotically flat and hence one cannot define charges for these objects. Nevertheless, one expects a relation, be it not 11 , between the BPS conditions of the different non-standard branes and the central charges. Indeed, for branes with two transverse directions, i.e. "defect branes", it has been found that this relation is always 1-2, i.e., each BPS condition corresponds to two defect branes [23]. These two defect branes are related by an S-duality to each other.

It is instructive to consider, as an example, the half-supersymmetric 7branes of IIB string theory and the central charges of the 10D IIB superalgebra. This algebra has a single 3 -form central charge $Z_{a b c}$ which is a singlet under the $R$-symmetry group $S O(2)$. On the one hand there is a 1-1 relation between this central charge and the D3-brane which is a singlet under the U-duality group $S L(2, \mathbb{R})$. Alternatively, one may consider the dual central charge $\tilde{Z}_{a_{1} \cdots a_{7}}$ and its relation to the 7 -branes of IIB string theory.

To be more specific, consider a 7 -brane extended in the directions $x^{1} \ldots x^{7}$. We define the complex transverse coordinate $z=x^{8}+i x^{9}$. Since we consider supersymmetric solutions the Killing spinor equations must be satisfied. The most general solution to these equations is given by $[55,56]$

$$
\begin{aligned}
d s^{2} & =-d t^{2}+d \vec{x}_{7}^{2}+(\operatorname{Im} \tau)|f|^{2} d z d \bar{z} \\
\tau & =\tau(z), \quad f=f(z) \\
\epsilon & =(f / \bar{f})^{1 / 4} \epsilon_{0}
\end{aligned}
$$

where $\tau=\chi+i e^{-\phi}$ is the axion-dilaton, $f(z)$ is a holomorphic function 
and $\epsilon_{0}$ is a constant spinor which satisfies $\gamma_{\underline{z}^{*}} \epsilon_{0}=0 .{ }^{12}$ Under $S L(2, \mathbb{R})$ the holomorphic functions $\tau(z), f(z)$ and $\epsilon(z)$ transform as

$$
\tau \rightarrow \Lambda \tau \equiv \frac{a \tau+b}{c \tau+d}, \quad f(z) \rightarrow(c \tau(z)+d) f(z), \quad \epsilon \rightarrow e^{i \varphi} \epsilon,
$$

where

$$
\Lambda=\left(\begin{array}{ll}
a & b \\
c & d
\end{array}\right) \in S L(2, \mathbb{R}) \quad \text { and } \quad \varphi=\frac{1}{2} \arg (c \tau+d) .
$$

More precisely, this means that $\tau(z)$ transforms under $P S L(2, \mathbb{R})$ and that $\epsilon(z)$ transforms under the double cover of $S L(2, \mathbb{R})$.

From the general supersymmetric configuration (5.1) we deduce that all solutions satisfy the following $S L(2, \mathbb{R})$-invariant BPS condition:

$$
\gamma_{\underline{z}^{*}} \epsilon=0
$$

From [23] we know that there are two elementary supersymmetric 7-branes. Using real notation $S O(2,1) \simeq S L(2, \mathbb{R})$ they correspond to the two lightcone directions of $S O(2,1)$. These branes are the D7-brane and its S-dual. This confirms the two-fold degeneracy of the BPS condition (5.4) mentioned in [23]. From the general analysis above we deduce that there is a third halfsupersymmetric 7-brane solution, corresponding to the third non-lightcone direction, that is not elementary, i.e. it has no supersymmetric WZ term. This 7-brane solution describes a threshold bound state of a D7-brane and an S-dual D7-brane. The 10D 7-brane situation is generic for all defect branes in $D \leq 10$ dimensions [23]. There are always twice as many defect branes as central charges since each defect brane and its S-dual have the same BPS projection operator.

We now wish to investigate whether a similar, not necessarily 1 -1, relation like we just found for the defect branes also holds between the central charges of the algebras with 32 supercharges and the elementary domain walls studied in this work. The central charges corresponding to supersymmetric domain walls are the duals $Z_{a_{1} \cdots a_{D-2}}$ of the 2-form central charges $Z_{a b}$ for $4 \leq D \leq 10$ dimensions while they are the 1 -form central charges $Z_{a}$ for $D=3$ dimensions. These central charges transform as representations of the

\footnotetext{
${ }^{12}$ We use complex notation in which $\epsilon$ can be written as $\epsilon=\epsilon_{1}+i \epsilon_{2}$ where $\epsilon_{1}$ and $\epsilon_{2}$ are two Majorana-Weyl spinors. We take the chirality of $\epsilon$ to be negative, i.e. $\gamma_{11} \epsilon=-\epsilon$. The underbar in $\underline{z}$ indicates that this is a flat index.
} 
$R$-symmetry group, see Table 9 , and we study them first for the standard 2and 1-branes that couple to them.

We expect a 1-1 relation between these central charges and branes with three or more transverse directions. We wish to verify this 1-1 relation by comparing, for each dimension, the central charges with the corresponding 2branes in $D \geq 6$. Starting with 10D we note that in the 10D IIA theory there is a single 2-form central charge and, correspondingly, a single D2-brane. In 9D the relevant brane is the D2-brane compactified over one of its transverse directions. In $8 \mathrm{D}$ there is a doublet of Dirichlet 2-branes which transform as a chiral spinor representation of the T-duality group $S L(2, \mathbb{R}) \times S L(2, \mathbb{R})$. In $7 \mathrm{D}$ there is a 4 -component $\mathrm{T}$-duality spinor of Dirichlet 2-branes and a singlet solitonic 2-brane. The second singlet given in Table 9 is a KaluzaKlein monopole. ${ }^{13}$ This adds up to a total of 6 branes corresponding to the 62 -form central charges indicated in the table. In 6D there is a 8-component T-duality spinor of Dirichlet 2-branes and a 8-component T-duality vector of solitonic 2-branes adding up to a total of 16 branes corresponding to the 162 -form central charges in 6D.

We now consider the relation between the 2-form charges and the 2branes for $D=5$ and $D=4$, as well as the relation between 1-form central charges and 1-branes in 3D. In 5D 2-branes have two transverse directions, i.e. they are defect branes. We therefore expect a 1-2 relation in this case. Indeed, there is a 16-component chiral T-duality spinor of Dirichlet 2-branes, a 40-dimensional orbit of solitonic 2-branes within the 45-dimensional adjoint representation of the T-duality group and a 16-component anti-chiral Tduality spinor of 2-branes with $\alpha=-3$. This adds up to a total number of 72 defect branes which, as expected, is twice the number of 5D 2-form central charges. In 4D 2-branes are domain walls and their total number is indicated in the last column of Table 9. Finally, in 3D domain walls are 1-branes and their total number is also given in the last column.

Returning to domain walls we can read off from Table 9 some general patterns although they are not as clean as in the case of the defect branes. We observe that for $5 \leq D \leq 10$ dimensions the number $n_{\mathrm{DW}}$ of supersymmetric domain walls with a worldvolume vector multiplet is $d+1$ times the number

\footnotetext{
${ }^{13} \mathrm{As}$ far as the central charge and BPS condition is concerned a KK monopole in $D$ dimensions behaves as a $(D-5)$-brane, i.e. in $D=7$ dimensions it behaves as a 2-brane.
} 
$n_{\mathrm{Z}}$ of corresponding 2 -form central charges, i.e. ${ }^{14}$

$$
n_{\mathrm{DW}}=(d+1) n_{\mathrm{Z}}, \quad 5 \leq D \leq 10 .
$$

Here, $d=10-D$. For $D=4$ and $D=3$ the relations are $n_{\mathrm{DW}}=8 n_{\mathrm{Z}}$ and $n_{\mathrm{DW}}=16 n_{\mathrm{Z}}$, respectively. Below we will discuss, for each dimension $6 \leq D \leq 10$ separately, starting with ten dimensions, how this degeneracy fits with our results on the embedding tensor obtained in section 3 .

$D=10$ : This case has been discussed extensively in the introduction. There is a single mass parameter $m$ which is a massive deformation (without any gauging) of IIA supergravity. The D8-brane is the half-supersymmetric domainwall solution of this massive IIA supergravity theory. Its BPS condition corresponds to the dual of the 2-form central charge in the IIA supersymmetry algebra. In this case we find a 1-1 relation between the dual of the 2-form central charge and the half-supersymmetric domain wall of the theory.

$D=9$ : In the previous section we have seen that the $\Theta^{i}(i=1,2,3)$ gaugings allow two elementary half-supersymmetric domain-wall solutions with the same BPS condition (4.1). These solutions correspond to the two lightcone directions of $S L(2, \mathbb{R}) \simeq S O(2,1)$. Comparing with Table 9 we conclude that there is a two-fold degeneracy: there are 2 half-supersymmetric elementary domain walls corresponding to the single 7 -form dual of the 2 -form central charge.

$D=8$ : In subsection 3.2 we found that there are three different minimal gaugings. Each domain wall corresponding to these three minimal gaugings has the same BPS condition. This leads to the three-fold degeneracy of the 6 -form dual of the 2-form central charge. Given the doublet of 2-form central charges given in Table 9, this leads to a total of 6 elementary halfsupersymmetric domain walls.

$D=7$ : In the previous sections we have seen that there are $20 \subset \overline{\mathbf{4 0}}$ elementary domain walls with vector multiplets and $5 \subset \overline{\mathbf{1 5}}$ elementary domain

\footnotetext{
${ }^{14}$ Note that in $7 \mathrm{D}$ the central charges occur in two different representations. This is related to the fact that in 7D there are two types of domain walls, with a different worldvolume content. There are 20 domain walls with a worldvolume vector multiplet for which eq. (5.5) applies, i.e. $n_{\mathrm{DW}}=4 n_{\mathrm{Z}}$ and there are five domain walls with a worldvolume tensor multiplet for which we have $n_{\mathrm{DW}}=5 n_{\mathrm{Z}}$.
} 


\begin{tabular}{|c|c|c|c|c|c|}
\hline$D$ & $H$ & $n=1$ & $n=2$ & 2 -branes & $n_{\text {DW }}$ \\
\hline IIA & 1 & & $\mathbf{1}$ & $\mathbf{1}_{-1}$ & 1 \\
\hline 9 & $S O(2)$ & & $\mathbf{1}$ & $\mathbf{1}_{-1}$ & 2 \\
\hline 8 & $S O(3) \times S O(2)$ & & $(\mathbf{1}, \mathbf{2})$ & $(\mathbf{1}, \mathbf{2})_{-1}$ & 6 \\
\hline 7 & $S p(4)$ & & $\mathbf{5}+\mathbf{1}$ & $\left(\mathbf{4}_{-1}+\mathbf{1}_{-2}\right)+1$ & $20+5$ \\
\hline 6 & $S p(4) \times S p(4)$ & & $(\mathbf{4}, \mathbf{4})$ & $\left(\mathbf{8}_{\mathrm{S}}\right)_{-1}+\left(\mathbf{8}_{\mathrm{C}}\right)_{-2}$ & 80 \\
\hline 5 & $S p(8)$ & & $\mathbf{3 6}$ & $\mathbf{1 6}_{-1}+40 \subset \mathbf{4 5}_{-2}+\overline{\mathbf{1 6}}_{-3}$ & 216 \\
\hline 4 & $S U(8)$ & & $\mathbf{3 6}^{+}+\overline{\mathbf{3 6}}^{-}$ & & 576 \\
\hline 3 & $S O(16)$ & $\mathbf{1 3 5}$ & & & 2160 \\
\hline
\end{tabular}

Table 9: This table indicates the 1-form and 2-form central charges that are related to supersymmetric domain walls. The number $n$ in the top row indicates the rank of the central charge. The $R$-symmetry group $H$ is indicated in the second column. The next two columns indicate the representations of $H$ according to which the 1-form and 2-form central charges transform. The fifth column gives the T-duality representations of the 2-branes (not the domain walls) associated to the 2-form central charges. The sub-index indicates the $\alpha$-value of these branes. The singlet in $7 D$, without a sub-index, corresponds to a Kaluza-Klein monopole. We have left the entries for the $4 D$ and $3 D$ cases empty since $4 D$ 2-branes and $3 D$ 1-branes are domain walls and as such are represented in the last column. This last column gives the total number of half-supersymmetric domain walls corresponding to the duals of the 1-form and 2-form central charges. Their $\alpha$-values are given in Table 3.

walls with tensor multiplets. From Table 9 we deduce that the vector domain walls have degeneracy 4 while the tensor domain walls have degeneracy 5 . From our general analysis it is easy to see why.

Consider first the vector domain walls. They correspond to minimal gaugings that are generated by the embedding tensor $\Theta^{M N, P}$. In subsection 3.3 we found that there are $4 \times 5$ minimal gaugings corresponding to this embedding tensor. The 5 in $4 \times 5$ corresponds to the last $S L(5, \mathbb{R})$ index of $\Theta^{M N, P}$. This direction is similar to the quintet of membranes that couple to the 3 -form potentials $A_{3 M}$. In the case of the membranes this leads to a single orbit of $\left(n_{1}, \ldots, n_{5}\right)$ membranes and a 1-1 relation between membranes and 2-form 
central charges. In the case of the domain walls we have an extra direction corresponding to the first two indices of $\Theta^{M N, P}$. This extra direction leads to 4 domains walls having the same BPS condition. This explains the four-fold degeneracy of the vector domain walls.

We next consider the tensor domain walls. We found in subsection 3.3 that in this case there are 5 different minimal gaugings leading to 5 elementary domain walls which have the same BPS condition. This leads to the 5 -fold degeneracy of the tensor domain walls.

$D=6$ : One can easily guess how the 5 -fold degeneracy of the 80 elementary domain walls with respect to the 16 central charges arises in six dimensions. The $(D-1)$-forms in six dimensions belong to the 144 representation of $S O(5,5)$, which is the vector-spinor representation. Introducing lightlike directions $n \pm, n=1, \ldots, 5$, one can always choose a basis of Gamma matrices in $S O(5,5)$ such that the highest-weight orbit of the $\mathbf{1 4 4}$ is such that for each of the 16 spinor components only one of the two lightlike directions $n+$ and $n$ - survives, for any $n$ (see the appendix of [9]). This indeed leads to $16 \times 5=80$ elementary domain walls. The 5 in the $16 \times 5$ product is the degeneracy: for each of the 16 spinor directions, the branes corresponding to the 5 different non-vanishing lightlike directions lead to the same BPS condition.

One can analyze in a similar way the lower dimensional cases. We leave this as an open project here.

\subsection{BPS conditions and $E_{11}$}

We can use the knowledge of the roots $\alpha$ corresponding to supersymmetric domain walls to also determine the Killing spinor conditions. This rests on the known correspondence between the variation of the gravitino and the $K\left(E_{10}\right)$ Dirac operator $[57,58,59]$. We explain this again in the $D=7$ example that was already treated in the previous section.

From an $E_{11}$ perspective the six-forms in the $\overline{\mathbf{1 5}} \oplus \overline{\mathbf{4 0}}$ belong to generators that can be conveniently described in the $G L(11, \mathbb{R})$ decomposition of

$E_{11}$. This decomposition gives an eleven-dimensional origin to the various six-forms. More precisely, they come from the following mixed symmetry 
generators of $E_{11}[16]$

$$
\underbrace{E^{a_{1} \ldots a_{6}}}_{\ell=2}, \underbrace{E^{a_{1} \ldots a_{8}, b}}_{\ell=3}, \underbrace{E^{a_{1} \ldots a_{9}, b_{1} b_{2} b_{3}}, E^{a_{1} \ldots a_{10}, b, c}}_{\ell=4}, \underbrace{E^{a_{1} \ldots a_{10}, b_{1} \ldots b_{4}, c}}_{\ell=5} .
$$

Here, we have also indicated the $G L(11, \mathbb{R})$ level $[41,60]$ in the decomposition. The notation is such that all indices in one block (with the same letter) are antisymmetric. These are irreducible representations so that antisymmetrization including one complete index block and any index from a block to the right gives zero. All these generators give rise to six-forms by putting a sufficient number of indices in the same direction. Putting the maximum number of indices identical will give real roots. This results in the following number of real roots (三 number of supersymmetric domain walls) in these generators written in terms of $G L(11, \mathbb{R})$ irreducibles

\begin{tabular}{|c|c|c|}
\hline Generator & contains \#(6-forms) & \#(real roots) \\
\hline$E^{a_{1} \ldots a_{6}}:$ & 1 & 1 \\
\hline$E^{a_{1} \ldots a_{8}, b}:$ & 24 & $12=4 \times 3$ \\
\hline$E^{a_{1} \ldots a_{9}, b_{1} b_{2} b_{3}}:$ & 16 & 4 \\
\hline$E^{a_{1} \ldots a_{10}, b, c}:$ & 10 & 4 \\
\hline$E^{a_{1} \ldots a_{10}, b_{1} \ldots b_{4}, c}:$ & 4 & $4=4 \times 1$ \\
\hline
\end{tabular}

(The Romans mass is contained in the fourth generator $(5.7 \mathrm{~d})$, see e.g. [61, $62,63]$.$) The generators in (5.7 \mathrm{a})$ and $(5.7 \mathrm{c})$ are the ones that belong to the five supersymmetric (tensor) domain walls in the $\overline{\mathbf{1 5}}$, the remaining ones give 20 supersymmetric (vector) domain walls from the $\overline{\mathbf{4 0}}$.

We will now use $K\left(E_{11}\right)$ to determine the supersymmetries that are preserved by the various branes in terms of their projectors. For the generators $(5.7)$ one can compute the action of the associated $K^{*}\left(E_{11}\right)$ generator $J=\left(E-(E)^{\#}\right) / 2$ on the 32-component supersymmetry parameter in the Dirac operator $[57,58,59,64]$. This results in

\begin{tabular}{|c|c|c|}
\hline Generator & Projector & $\#($ real roots $)$ \\
\hline$J^{a_{1} \ldots a_{6}} \rightarrow$ & $\Gamma^{012345}$ & 1 \\
\hline$J^{a_{1} \ldots a_{8}, b} \rightarrow$ & $\Gamma^{012345} \Gamma^{i}$ & $4 \times 3$ \\
\hline$J^{a_{1} \ldots a_{9}, b_{1} b_{2} b_{3}} \rightarrow$ & $\Gamma^{012345}$ & 4 \\
\hline$J^{a_{1} \ldots a_{10}, b, c} \rightarrow$ & $\Gamma^{012345} \Gamma^{78910}$ & 4 \\
\hline$J^{a_{1} \ldots a_{10}, b_{1} \ldots b_{4}, c} \rightarrow$ & $\Gamma^{012345} \Gamma^{i}$ & $4 \times 1$ \\
\hline
\end{tabular}


Here, the gamma matrices are those of eleven dimensions which are of size $32 \times 32$. The index $i=7,8,9,10$ labels one of the four compact directions and we aligned the elementary brane along the directions $0 \ldots 5$, leaving 6 as the transverse direction. We see that there are different types of projectors. The ones in (5.8a) and (5.8c) are the same, leading to a five-fold degeneracy of the BPS condition for the tensor brane. All five elementary tensor branes couple to the same central charge.

For the vector branes, there are in total five different projectors. Four are of the form $\Gamma^{012345} \Gamma^{i}$ with $i=7,8,9,10$, and one is of the form $\Gamma^{012345} \Gamma^{78910}$. Each projection condition is four-fold degenerate. In the latter case (5.8d) this is obvious, in the former case one has to combine three contributions from $(5.8 \mathrm{~b})$ with one from $(5.8 \mathrm{e})$. The BPS projectors corresponding to the intersecting brane configuration presented in eq. (4.12) are given by (5.8a) and (5.8b). These projectors are not orthogonal and combining them yields a projector on a subspace for a $1 / 4$-BPS state.

From the form of the root vectors and the $K^{*}\left(E_{10}\right)$ Dirac operator it is also possible to obtain the rescaling of the Killing spinor that enters the Killing spinor equation. Generally, the Killing spinor is

$$
\epsilon=H^{p_{\perp} / 2} \epsilon_{0},
$$

where $p_{\perp}$ is the component of the root (in a metric basis like in (4.11)) along the transverse direction.

A similar analysis can in principle be carried out in all dimensions in order to determine the supersymmetries preserved by the various domain walls. The same logic works for other branes than domain walls as well.

\section{Conclusions}

In this paper we have analyzed the connection between half-supersymmetric domain walls and deformed maximal supergravity theories. One of the main results was that elementary supersymmetric domain walls exist only in minimally deformed supergravities. An elementary domain wall was defined as a domain wall whose dynamics can be described by a supersymmetric worldvolume action, while a minimal deformed supergravity was defined as a supergravity in which the rearrangement of degrees of freedom, which takes place after the deformation has been turned on, is minimal in the sense that the minimal number of fields are involved in this rearrangement. Both the 
elementary domain walls and the minimal gaugings can be characterized in terms of highest-weight orbits under U-duality. We found that there are many more (non-minimal) gauged supergravity theories that admit supersymmetric domain-wall solutions. These non-elementary domain walls correspond to bound states of the elementary ones. There exist different types of bound states. The ones that preserve half supersymmetry, like the elementary domain walls themselves, are called bound states at threshold. The ones that preserve less supersymmetry are non-threshold bound states. The dynamics of these bound states, threshold and non-threshold, can not be described by a standard supersymmetric worldvolume action.

We have elucidated the connection between the elementary domain walls and the central charges in the supersymmetry algebra. We found that the relation is many-to-one in contrast to what happens for branes of codimension three or higher. This extends an earlier result of ours where we found a twoto-one relation between defect branes, i.e., branes of codimenion 2 , and the central charges of the supersymmetry algebra. We have explicitly shown the degeneracy of the BPS conditions at the level of the domain-wall solutions. The number of central charges times the degeneracy of the BPS conditions equals the number of vector minimal gaugings. This degeneracy, for vector domain walls, is $d+1$ for dimensions $5 \leq D \leq 10$, while it is 8 in $4 \mathrm{D}$ and 16 in $3 \mathrm{D}$. The tensor minimal gaugings in $7 \mathrm{D}$, i.e. the ones that lead to tensor domain-wall solutions, are special in the sense that they lead to a massive self-dual 3-form. For these domain walls the degeneracy is 5. Our results could be rephrased in terms of the $E_{11}$ Kac-Moody algebra.

The fact that there are many domain walls associated to a given central charge, and therefore to a given BPS projection, explains the fact that there are threshold bound states, preserving the same amount of supersymmetry as the elementary domain walls. Indeed, a bound state of two elementary domain walls both satisfying the same BPS projection does not break supersymmetry any further. Non-threshold bound states, instead, are bound states of elementary domain walls satisfying different BPS conditions.

The domain-wall solutions we presented were only local solutions and do not necessarily have finite energy. Finding rules for a proper periodic arrangement with orientifolds is an interesting question, as would be the application of the solutions in the domain wall/cosmology correspondence mentioned in the introduction. Furthermore, the application to (A)dS spaces might prove fruitful since gauged supergravities typically have rather complicated potentials also allowing for AdS vacua. 
Finally, it would be of interest to extend our analysis to cases where the original supergravity theory has less than maximal supersymmetry. We hope to come back to these and other interesting issues in the future.

\section{Acknowledgements}

A large part of this work was completed at the Isaac Newton Institute for Mathematical Sciences (Cambridge). We thank the organizers of the "Mathematics and Applications of Branes in String and M-theory" programme for their hospitality and financial support. We wish to thank Diederik Roest and Henning Samtleben for useful discussions.

\section{References}

[1] N. Kaloper, "Bent domain walls as brane worlds," Phys. Rev. D 60 (1999) 123506 [hep-th/9905210].

[2] K. Skenderis and P. K. Townsend, "Gravitational stability and renormalization group flow," Phys. Lett. B 468 (1999) 46 [hep-th/9909070].

[3] K. Skenderis, P. K. Townsend and A. Van Proeyen, "Domainwall/cosmology correspondence in adS/dS supergravity," JHEP 0708 (2007) 036 [arXiv:0704.3918 [hep-th]]; K. Skenderis and P. K. Townsend, "Pseudo-Supersymmetry and the Domain-Wall/Cosmology Correspondence," J. Phys. A A 40 (2007) 6733 [hep-th/0610253]; ibid., "Hidden supersymmetry of domain walls and cosmologies," Phys. Rev. Lett. 96 (2006) 191301 [hep-th/0602260].

[4] L. J. Romans, "Massive N=2a Supergravity in Ten-Dimensions," Phys. Lett. B 169 (1986) 374.

[5] E. Bergshoeff, M. de Roo, M. B. Green, G. Papadopoulos and P. K. Townsend, "Duality of type II 7 branes and 8 branes," Nucl. Phys. B 470 (1996) 113 [hep-th/9601150].

[6] E. A. Bergshoeff, M. de Roo, S. F. Kerstan, T. Ortín and F. Riccioni, "IIA ten-forms and the gauge algebras of maximal supergravity theories," JHEP 0607 (2006) 018 [hep-th/0602280]; E. A. Bergshoeff, J. Har- 
tong, P. S. Howe, T. Ortín and F. Riccioni, "IIA/IIB Supergravity and Ten-forms," JHEP 1005 (2010) 061 [arXiv:1004.1348 [hep-th]].

[7] E. Bergshoeff, P. M. Cowdall and P. K. Townsend, "Massive IIA supergravity from the topologically massive D2-brane," Phys. Lett. B 410 (1997) 13 [hep-th/9706094]; E. Bergshoeff, Y. Lozano and T. Ortin, "Massive branes," Nucl. Phys. B 518 (1998) 363 [hep-th/9712115];

[8] E. A. Bergshoeff and F. Riccioni, "D-Brane Wess-Zumino Terms and U-Duality," JHEP 1011 (2010) 139 [arXiv:1009.4657 [hep-th]].

[9] E. A. Bergshoeff and F. Riccioni, "String Solitons and T-duality," JHEP 1105 (2011) 131 [arXiv:1102.0934 [hep-th]].

[10] E. A. Bergshoeff and F. Riccioni, "The D-brane U-scan," arXiv:1109.1725 [hep-th].

[11] E. A. Bergshoeff, A. Marrani and F. Riccioni, "Brane orbits," Nucl. Phys. B 861 (2012) 104 [arXiv:1201.5819 [hep-th]].

[12] A. Kleinschmidt, "Counting supersymmetric branes," JHEP 1110 (2011) 144 [arXiv:1109.2025 [hep-th]].

[13] P. C. West, "E(11) and M theory," Class. Quant. Grav. 18 (2001) 4443 [hep-th/0104081].

[14] H. Nicolai and H. Samtleben, "Maximal gauged supergravity in three-dimensions," Phys. Rev. Lett. 86 (2001) 1686 [hep-th/0010076]; H. Nicolai and H. Samtleben, "Compact and noncompact gauged maximal supergravities in three-dimensions," JHEP 0104 (2001) 022 [hep-th/0103032]; B. de Wit, H. Samtleben and M. Trigiante, "On Lagrangians and gaugings of maximal supergravities," Nucl. Phys. B 655 (2003) 93 [hep-th/0212239]; B. de Wit, H. Samtleben and M. Trigiante, "The Maximal $\mathrm{D}=5$ supergravities," Nucl. Phys. B 716 (2005) 215 [hep-th/0412173]; B. de Wit and H. Samtleben, "Gauged maximal supergravities and hierarchies of nonabelian vector-tensor systems," Fortsch. Phys. 53 (2005) 442 [hep-th/0501243].

[15] B. de Wit, H. Nicolai and H. Samtleben, "Gauged Supergravities, Tensor Hierarchies, and M-Theory," JHEP 0802 (2008) 044 [arXiv:0801.1294 [hep-th]]. 
[16] F. Riccioni and P. C. West, "The E(11) origin of all maximal supergravities," JHEP 0707 (2007) 063 [arXiv:0705.0752 [hep-th]].

[17] E. A. Bergshoeff, I. De Baetselier and T. A. Nutma, "E(11) and the embedding tensor," JHEP 0709 (2007) 047 [arXiv:0705.1304 [hep-th]].

[18] P. Henry-Labordere, B. Julia and L. Paulot, "Borcherds symmetries in M theory," JHEP 0204 (2002) 049 [hep-th/0203070]; M. Henneaux, B. L. Julia and J. Levie, " $E_{11}$, Borcherds algebras and maximal supergravity," JHEP 1204 (2012) 078 [arXiv:1007.5241 [hep-th]].

[19] J. Palmkvist, "Tensor hierarchies, Borcherds algebras and E11," JHEP 1202 (2012) 066 [arXiv:1110.4892 [hep-th]].

[20] J. Greitz and P. S. Howe, JHEP 1107 (2011) 071 [arXiv:1103.2730 [hepth]].

[21] P. M. Cowdall, H. Lu, C. N. Pope, K. S. Stelle and P. K. Townsend, "Domain walls in massive supergravities," Nucl. Phys. B 486 (1997) 49 [hep-th/9608173].

[22] J. A. de Azcarraga, J. P. Gauntlett, J. M. Izquierdo and P. K. Townsend, "Topological Extensions of the Supersymmetry Algebra for Extended Objects," Phys. Rev. Lett. 63 (1989) 2443.

[23] E. Bergshoeff, T. Ortín and F. Riccioni, "Defect Branes," Nucl. Phys. B 856, 210 (2012) [arXiv:1109.4484 [hep-th]].

[24] E. A. Bergshoeff, M. de Roo, S. F. Kerstan and F. Riccioni, "IIB supergravity revisited," JHEP 0508 (2005) 098 [hep-th/0506013].

[25] F. Englert and L. Houart, "G+++ invariant formulation of gravity and M theories: Exact BPS solutions," JHEP 0401 (2004) 002 [hep-th/0311255].

[26] F. Englert and L. Houart, "G+++ invariant formulation of gravity and M-theories: Exact intersecting brane solutions," JHEP 0405 (2004) 059 [hep-th/0405082].

[27] P. C. West, "The IIA, IIB and eleven-dimensional theories and their common E(11) origin," Nucl. Phys. B 693 (2004) 76 [hep-th/0402140]. 
[28] P. P. Cook and P. C. West, "G+++ and brane solutions," Nucl. Phys. B 705 (2005) 111 [hep-th/0405149].

[29] L. Houart, A. Kleinschmidt and J. Lindman Hornlund, "An M-theory solution from null roots in E11," JHEP 1101 (2011) 154 [arXiv:1101.2816 [hep-th]].

[30] N. A. Obers and B. Pioline, "U duality and M theory," Phys. Rept. 318 (1999) 113 [hep-th/9809039].

[31] H. Samtleben and M. Weidner, "The Maximal D=7 supergravities," Nucl. Phys. B 725 (2005) 383 [hep-th/0506237].

[32] E. Bergshoeff, T. de Wit, U. Gran, R. Linares and D. Roest, "(Non)Abelian gauged supergravities in nine-dimensions," JHEP 0210 (2002) 061 [hep-th/0209205].

[33] J. J. Fernandez-Melgarejo, T. Ortín and E. Torrente-Lujan, "The general gaugings of maximal d=9 supergravity," JHEP 1110 (2011) 068 [arXiv:1106.1760 [hep-th]].

[34] M. de Roo, G. Dibitetto and Y. Yin, "Critical points of maximal D=8 gauged supergravities," JHEP 1201 (2012) 029 [arXiv:1110.2886 [hepth]].

[35] G. Dibitetto, J. J. Fernandez-Melgarejo, D. Marques and D. Roest, "Duality orbits of non-geometric fluxes," arXiv:1203.6562 [hep-th].

[36] N. Alonso Alberca, E. Bergshoeff, U. Gran, R. Linares, T. Ortín and D. Roest, "Domain walls of $\mathrm{D}=8$ gauged supergravities and their $\mathrm{D}=$ 11 origin," JHEP 0306 (2003) 038 [hep-th/0303113].

[37] E. Bergshoeff, U. Gran, R. Linares, M. Nielsen, T. Ortín and D. Roest, "The Bianchi classification of maximal D $=8$ gauged supergravities," Class. Quant. Grav. 20 (2003) 3997 [hep-th/0306179].

[38] A. Salam and E. Sezgin, “d = 8 supergravity,” Nucl. Phys. B 258 (1985) 284.

[39] M. de Roo, D. B. Westra and S. Panda, "Gauging CSO groups in N=4 Supergravity," JHEP 0609 (2006) 011 [hep-th/0606282]. 
[40] E. Bergshoeff, U. Gran and D. Roest, "Type IIB seven-brane solutions from nine-dimensional domain walls," Class. Quant. Grav. 19 (2002) 4207 [hep-th/0203202].

[41] T. Damour, M. Henneaux and H. Nicolai, "E(10) and a 'small tension expansion' of M theory," Phys. Rev. Lett. 89 (2002) 221601 [hep-th/0207267].

[42] A. Keurentjes, "E(11): Sign of the times," Nucl. Phys. B 697 (2004) 302 [hep-th/0402090].

[43] A. Keurentjes, "Time-like T duality algebra," JHEP 0411 (2004) 034 [hep-th/0404174].

[44] T. Damour and H. Nicolai, "Eleven dimensional supergravity and the $\mathrm{E}(10) / \mathrm{K}(\mathrm{E} 10)$ sigma-model at low A(9) levels," hep-th/0410245.

[45] A. Kleinschmidt and H. Nicolai, "E(10) cosmology," JHEP 0601 (2006) 137 [hep-th/0511290].

[46] P. P. Cook, "Exotic E(11) branes as composite gravitational solutions," Class. Quant. Grav. 26 (2009) 235023 [arXiv:0908.0485 [hep-th]].

[47] L. Houart, A. Kleinschmidt and J. Lindman Hornlund, "Some Algebraic Aspects of Half-BPS Bound States in M-Theory," JHEP 1003 (2010) 022 [arXiv:0911.5141 [hep-th]].

[48] F. Englert, M. Henneaux and L. Houart, "From very-extended to overextended gravity and M-theories," JHEP 0502 (2005) 070 [hep-th/0412184].

[49] F. Englert, L. Houart, A. Kleinschmidt, H. Nicolai and N. Tabti, "An E(9) multiplet of BPS states," JHEP 0705 (2007) 065 [hep-th/0703285].

[50] T. Damour, S. de Buyl, M. Henneaux and C. Schomblond, "Einstein billiards and overextensions of finite dimensional simple Lie algebras," JHEP 0208 (2002) 030 [hep-th/0206125].

[51] F. Englert, L. Houart, A. Taormina and P. C. West, "The Symmetry of M theories," JHEP 0309 (2003) 020 [hep-th/0304206]. 
[52] P. P. Cook, "Exotic E(11) branes as composite gravitational solutions," Class. Quant. Grav. 26 (2009) 235023 [arXiv:0908.0485 [hep-th]].

[53] L. Houart, A. Kleinschmidt and J. L. Hornlund, "Some Algebraic Aspects of Half-BPS Bound States in M-Theory," JHEP 1003 (2010) 022 [arXiv:0911.5141 [hep-th]].

[54] P. K. Townsend, "M theory from its superalgebra," In Cargese 1997, Strings, branes and dualities 141-177 [hep-th/9712004].

[55] B. R. Greene, A. D. Shapere, C. Vafa and S. -T. Yau, "Stringy Cosmic Strings and Noncompact Calabi-Yau Manifolds," Nucl. Phys. B 337 (1990) 1.

[56] E. A. Bergshoeff, J. Hartong, T. Ortín and D. Roest, "Seven-branes and Supersymmetry," JHEP 0702 (2007) 003 [hep-th/0612072].

[57] T. Damour, A. Kleinschmidt and H. Nicolai, "Hidden symmetries and the fermionic sector of eleven-dimensional supergravity," Phys. Lett. B 634 (2006) 319 [hep-th/0512163].

[58] S. de Buyl, M. Henneaux and L. Paulot, "Extended E(8) invariance of 11-dimensional supergravity," JHEP 0602 (2006) 056 [hep-th/0512292].

[59] T. Damour, A. Kleinschmidt and H. Nicolai, "K(E(10)), Supergravity and Fermions," JHEP 0608 (2006) 046 [hep-th/0606105].

[60] P. C. West, "Very extended E(8) and A(8) at low levels, gravity and supergravity," Class. Quant. Grav. 20 (2003) 2393 [hep-th/0212291].

[61] I. Schnakenburg and P. C. West, "Massive IIA supergravity as a nonlinear realization," Phys. Lett. B 540 (2002) 137 [hep-th/0204207].

[62] A. Kleinschmidt, I. Schnakenburg and P. C. West, "Very extended KacMoody algebras and their interpretation at low levels," Class. Quant. Grav. 21 (2004) 2493 [hep-th/0309198].

[63] M. Henneaux, E. Jamsin, A. Kleinschmidt and D. Persson, "On the E10/Massive Type IIA Supergravity Correspondence," Phys. Rev. D 79 (2009) 045008 [arXiv:0811.4358 [hep-th]]. 
[64] P. C. West, "E(11), SL(32) and central charges," Phys. Lett. B 575 (2003) 333 [hep-th/0307098]. 